\title{
Radon transform and pattern functions in quantum tomography
}

\author{
Alfred Wünsche \\ Arbeitsgruppe "Nichtklassische Strahlung" der Max-Planck-Gesellschaft \\ Rudower Chaussee 5, 12489 Berlin, Germany \\ e-mail:wuensche@photon.fta-berlin.de \\ tel.: (4930) 63923982 \\ fax: (4930) 63923990
}




\begin{abstract}
The two-dimensional Radon transform of the Wigner quasiprobability is introduced in canonical form and the functions playing a role in its inversion are discussed. The transformation properties of this Radon transform with respect to displacement and squeezing of states are studied and it is shown that the last is equivalent to a symplectic transformation of the variables of the Radon transform with the contragredient matrix to the transformation of the variables in the Wigner quasiprobability. The reconstruction of the density operator from the Radon transform and the direct reconstruction of its Fock-state matrix elements and of its normally ordered moments are discussed. It is found that for finite-order moments the integration over the angle can be reduced to a finite sum over a discrete set of angles. The reconstruction of the Fock-state matrix elements from the normally ordered moments leads to a new representation of the pattern functions by convergent series over even or odd Hermite polynomials which is appropriate for practical calculations. The structure of the pattern functions as first derivatives of the products of normalizable and nonnormalizable eigenfunctions to the number operator is considered from the point of view of this new representation.
\end{abstract}




\section{Introduction}

The Radon transform of the Wigner quasiprobability is closely related to the expectation values or densities formed with the eigenstates to the rotated canonical observables. They possess all properties of genuine probability densities and can be measured since recent time in quantum optics of the radiation field by homodyne detection [1-4]. The field of problems of the reconstruction of the density operator from such or similar data is called quantum tomography. There arises the problem of the reconstruction of the density operator or of related quantities as matrix elements of the density operator or ordered moments from a complete set of tomographic data or of their approximate and, in some sense, optimal reconstruction from a more or less incomplete set of tomographic data. The complete tomographic reconstruction of the density operator from the Radon transform of the Wigner quasiprobability was prepared, as sometimes happens with things in history, in a wondrous manner by a theoretical paper of Vogel and Risken [5] shortly before the experimental realisation of the corresponding measurements in quantum optics, although Vogel and Risken did not mention Radon transforms in this connection but speak about rotated quadrature phase. The transformation which is now called Radon transformation and which means the determination of field functions from their integrated values over some manifolds was introduced by Radon in 1917 but did not have a great impact on physics and also mathematics up to the fifties. Only, beginning from the sixties and prepared by mathematical papers as [6] and the requirements of different tomographic problems the Radon transformation and its inversion became interesting for a greater community that is reflected in monographs [7] and review articles [8].

The theoretical development in quantum tomography in the last years went in the direction to determine more directly physical relevant parameters of the density operator as, for example, its matrix elements in the Fock-state basis [9-21] or in the position representation [22, 23] or to determine directly the normally ordered moments from tomographic data [24, 25]. Other sets of measurable quantities as the displaced Fock-state matrix elements of the density operator with fixed displacement parameter and varied excitation numbers are investigated for its appropriateness to the reconstruction of the density operator [26]. Another direction is to study the influence of imperfect measurements onto the Radon transform of the Wigner quasiprobability. If this influence consists only in a smoothing of the Wigner quasiprobability and of its Radon transform resulting in its convolution with a Gaussian function then there is, in principle, no loss of information and one has to take one of the smoothed quasiprobabilities instead of the Wigner quasiprobability. This was taken into account in some of the first papers about reconstruction ( e.g., [14] ) and was prepared 
already by the paper of Vogel and Risken [5] starting from the rotated quadrature components of the $s$-orderd quasiprobabilities. Another problem arises when there is a real loss or incomplete knowledge of information. Then the reconstruction is no more unique and one needs a principle for an optimal reconstruction under such conditions. This problem was recently studied from a general point of view in [27] where the incomplete knowledge was called observation level and this could become important in quantum tomography in the next time. The necessary principle is Jaynes' principle of maximal entropy and the best choice for the entropy in quantum mechanics is, in our opinion, for some formal reasons the Von-Neumann entropy.

The direct reconstruction of the matrix elements of the density operator in the Fockstate basis initiated by D'Ariano, Macchiavello and Paris [9] leads to the integration over the angle and over the position of the rotated quadrature components multiplied by specific functions of the angle and of the position coordinate. These auxiliary functions split into simple angle-dependent phase functions and more complicated position-dependent functions which were called pattern functions $[12-14]$ and were calculated in a representation by the functions of the parabolic cylinder. Then it was found that these pattern functions can be represented as first derivatives of products of the normalizable eigenfunction with one nonnormalizable eigenfunction of the number operator to eigenvalues corresponding to the numbers in the considered Fock-state matrix elements [18]. The best way to recognize this special structure of the pattern functions is to consider the differential equations for the products of the eigenfunctions ( Hermite functions for the harmonic oscillator ) and to establish the corresponding orthogonality relations by standard methods of the theory of ordinary differential equations that means with the help of the adjoint equations [19, 20].

The usual restriction of the representation of the Radon transform in quantum optics to the dependence on two variables ( rotation angle and line coordinate ) is unfavourable when considering the transformation properties with respect to squeezing of the states which results in symplectic transformations of the arguments of the Wigner quasiprobability and of its Radon transform. Therefore, in [25] was chosen a more general concept for the Radon transform as the starting point and it was explicitly calculated this Radon transform for squeezed coherent states. The same more general concept was taken in [28, 29] and called symplectic tomography and since the Radon transform contains complete information about the quantum-mechanical state the basic equations of quantum mechanics were reformulated for the Radon transform. This has the advantage that one works directly with measurable quantities which can be considered as genuine probability densities, at least, for each separate choice of the angle.

In the present paper we introduce in section 2 the two-dimensional Radon transform, as it seems to us, in its most rational form which we call canonical form and consider its inversion where our intention is to clarify the meaning of some mathematical aspects which play a role in the inversion of two-dimensional Radon transforms and to give a new representations of this inversion. In section 3 we consider the transformation properties of the Wigner quasiprobability and of its Radon and Fourier transforms under displacement and squeezing transformations of initial states. The developed formulae, although highly technical and partially complicated, seem to us as very important for practical applications and theoretical 
calculations of the influence of squeezing that will be demonstrated for squeezed coherent states. Section 4 is devoted to the introduction and calculation of the pattern functions [1214] for the direct reconstruction of the Fock-state matrix elements from tomographic data and follows with weak deviations the main stream of considerations in the literature about quantum tomography but this section is also important as a preparation of the content of the next two sections. In section 5 we consider in a short form the direct reconstruction of the normally ordered moments from complete tomographic data without integration over the angle as recently published in our paper [25] and give some new formulae not represented there. Starting from the reconstruction of the density operator via the normally ordered moments we derive in section 6 an essentially new representation of the pattern functions for the reconstruction of the Fock-state matrix elements of the density operator in form of series over Hermite polynomials of even or odd order which is an alternative for the practical calculation of these functions to the existing representations by functions of the parabolic cylinder or by first derivatives of the product of the normalizable with a certain nonnormalizable eigenfunctions of the number operator in "position" representation. We find in this section a nonuniqueness of the pattern functions for the reconstruction of the Fock-state matrix elements. In section 7 we derive the specific structure of the pattern functions as derivatives of a product of a normalizable and a nonnormalizable wave function from the fourth-order differential equation for products of Hermite functions and its adjoint differential equation. Here we come again across with the mentioned nonuniqueness of the pattern functions. 


\section{Two-dimensional Radon and Fourier transforms and their inversion}

The Wigner quasiprobability is the best compromise in quantum mechanics for a phase-space description of a quantum-mechanical state in analogy to the phase-space description in classical mechanics and statistics [30-38]. We consider one field mode corresponding to one mechanical degree of freedom and use a pair of canonical coordinates $(q, p)$ or complex coordinates $\left(\alpha, \alpha^{*}\right)$ and corresponding pairs of canonical operators $(Q, P)$ or boson annihilation and creation operator $\left(a, a^{\dagger}\right)$ in the following way

$$
\alpha=\frac{q+i p}{\sqrt{2 \hbar}}, \quad \alpha^{*}=\frac{q-i p}{\sqrt{2 \hbar}}, \quad a=\frac{Q+i P}{\sqrt{2 \hbar}}, \quad a^{\dagger}=\frac{Q-i P}{\sqrt{2 \hbar}}, \quad \frac{i}{2} d \alpha \wedge d \alpha^{*}=\frac{d q \wedge d p}{2 \hbar} .
$$

In this section we consider the Radon transform of the Wigner quasiprobability its connection to the Fourier transform of the Wigner quasiprobability and the inversion of these transformations. All considerations of this section are not specific for the Wigner quasiprobability and one can exchange the symbol for the Wigner quasiprobability by the symbol for an arbitrary other function of two variables over a plane, for example, another quasiprobability and one obtains in this way the general properties of two-dimensional Radon transformations. Specific properties of the Wigner quasiprobability and its Radon and Fourier transforms are considered from section 3 on. We use here the representation by the real variables $(q, p)$. All these relations can be easily translated into a representation by complex variables.

The Radon transform $\breve{W}(u, v ; c)$ of the Wigner quasiprobability $W(q, p)$ can be defined in the following canonical form ( compare [6-8] )

$$
\breve{W}(u, v ; c) \equiv \int d q \wedge d p \delta(c-u q-v p) W(q, p) .
$$

The normalization of the Radon transform is closely connected to the normalization of the Wigner quasiprobability in the following way

$$
\int_{-\infty}^{+\infty} d c \breve{W}(u, v ; c)=\int d q \wedge d p W(q, p)=1 .
$$

Due to the relation

$$
\breve{W}(\mu u, \mu v ; \mu c)=\frac{1}{|\mu|} \breve{W}(u, v ; c), \quad \mu \in R
$$


where $\mu$ is an arbitrary real number this Radon transform depends effectively only on two continuous variables. The presence of the delta function under the two-dimensional integral in (2.2) restricts the integrations to the one-dimensional objects

$$
u q+v p=c .
$$

These are the equations for straight lines with $(u, v)$ as a normal vector to the lines and $c / \sqrt{u^{2}+v^{2}}$ as a measure for the orthogonal ( nearest ) distance of the line to the coordinate origin. However, this "oriented distance" can take on all real values contrary to positively definite distances. The coordinates $(u, v ; c)$ are homogeneous line coordinates in the dual plane to the $(q, p)$-plane. In case of the generalization of the Radon transform to $N$-dimensional spaces the corresponding $(N-1)$-dimensional objects over which the field is integrated are the $(N-1)$-dimensional hyperplanes but this will be not considered here. Due to Eq.(2.2) the full information of the Radon transform is already contained in $\breve{W}\left(\cos \varphi, \sin \varphi ; q_{\varphi}\right)$ where $(u, v)=(\cos \varphi, \sin \varphi)$ is now the normal unit vector to the straight lines. This reduced Radon transform is mostly denoted by $w\left(\varphi, q_{\varphi}\right)$ but we will see in the next section that such a notation, although shorter, is unfavourable for the discussion of the transformation properties of the Radon transform. We call the Radon transform as introduced in Eq.(2.2) the canonical form.

The Radon transform $\breve{W}(u, v ; c)$ is closely related to the Fourier transform $\tilde{W}(u, v)$ of the Wigner quasiprobability $W(q, p)$ which can be defined by

$$
\tilde{W}(u, v) \equiv \int d q \wedge d p \exp \{-i(u q+v p)\} W(q, p)
$$

with the inversion

$$
W(q, p)=\frac{1}{(2 \pi)^{2}} \int d u \wedge d v \exp \{i(u q+v p)\} \tilde{W}(u, v),
$$

and with the normalization

$$
\tilde{W}(0,0)=\int d q \wedge d p W(q, p)=1
$$

The relation of the Radon transform to the Fourier transform is given by

$$
\tilde{W}(u, v)=\int_{-\infty}^{+\infty} d c \exp (-i b c) \breve{W}\left(\frac{u}{b}, \frac{v}{b} ; c\right), \quad b \in R
$$

with arbitrary real numbers $b$ and its inversion is given by

$$
\breve{W}(u, v ; c)=\frac{1}{2 \pi} \int_{-\infty}^{+\infty} d b \exp (i b c) \tilde{W}(b u, b v) .
$$

The full inversion of the two-dimensional Radon transform can be made in two steps. The first step is the transition from the Radon transform to the Fourier transform and the second 
step is the inversion of the Fourier transform. If we introduce polar coordinates $(r, \varphi)$ instead of $(u, v)$ the following integral can be accomplished

$$
\begin{aligned}
\int_{-\infty}^{+\infty} d r|r| \exp (-i x r) & =i \frac{\partial}{\partial x}\left(\int_{0}^{+\infty} d r \exp (-i x r)-\int_{-\infty}^{0} d r \exp (-i x r)\right) \\
& =i \frac{\partial}{\partial x}\left(\frac{1}{i(x-i 0)}+\frac{1}{i(x+i 0)}\right) \\
& =2 \frac{\partial}{\partial x} \mathcal{P} \frac{1}{x}
\end{aligned}
$$

where $\mathcal{P}$ in front of $1 / x$ means that integrals over the singularity of this function must be taken in the sense of Cauchy's principal value. Then we find

$$
W(q, p)=\frac{r_{0}^{2}}{2 \pi^{2}} \int_{0}^{\pi} d \varphi \int_{-\infty}^{+\infty} d c\left(\frac{\partial}{\partial c} \mathcal{P} \frac{1}{c-r_{0}(q \cos \varphi+p \sin \varphi)}\right) \breve{W}\left(r_{0} \cos \varphi, r_{0} \sin \varphi ; c\right),
$$

with arbitrary real fixed $r_{0}$. The integral over $c$ can be represented after partial integration as the Hilbert transform of the derivative of the Radon transform with respect to the variable c. A more symmetrical way of the representation of the inversion formula can be obtained if first the integration over $c$ is made in the two-step inversion via the Fourier transform. One finds

$$
W(q, p)=\frac{1}{2 \pi^{2}} \int d u \wedge d v\left(\mathcal{P} \frac{1}{c-u q-v p}\right)^{(1)}|c| \breve{W}(u, v ; c), \quad c \in R,
$$

where the upper index (1) denotes the first derivative and where $c$ is an arbitrary real number.

It seems that the meaning of $(\partial / \partial x) \mathcal{P}(1 / x)$ is not very clear in the literature about quantum tomography. In particular, one cannot write $-\mathcal{P}\left(1 / x^{2}\right)$ as the result of this differentiation. Let us therefore discuss this more in detail. One has to consider $(\partial / \partial x) \mathcal{P}(1 / x)$ as a generalized function or linear functional and has to define all its derivatives as generalized derivatives in the sense of the theory of generalized functions. We introduce here the symbol $\mathcal{R}$ for the canonical regularization of a singular function (Gel'fand and Shilov [39] use the symbol k.r. in the Russian and $C R$ in the English edition for canonical regularization ). Canonical regularizations of different singular functions are synchronized in the sense that with respect to additions of functions, multiplication of functions by numbers and by wellbehaved smooth functions and differentiations the regularization symbol can be changed in its order with these operations. The canonical regularization of the singular function $1 / x$ is identical with the principal value $\mathcal{P}(1 / x)$. If $\varphi(x)$ denotes arbitrary basis functions then one can define $\mathcal{R}(1 / x)$ by the following linear functional ( we do not specify in this physical context the space of basis functions which must be sufficiently smooth, in particular, at $x=0$ and sufficiently decreasing in infinity together with their derivatives )

$$
\begin{aligned}
& \left(\mathcal{R} \frac{1}{x}, \varphi(x)\right) \equiv \int_{-\infty}^{+\infty} d x\left(\mathcal{R} \frac{1}{x}\right) \varphi(x) \equiv \int_{0}^{+\infty} d x \frac{\varphi(x)-\varphi(-x)}{x} \\
& \int_{-\infty}^{+\infty} d x\left(\mathcal{P} \frac{1}{x}\right) \varphi(x) \equiv \lim _{\varepsilon \rightarrow 0} \int_{|x| \geq \varepsilon} d x \frac{\varphi(x)}{x}, \quad \mathcal{R} \frac{1}{x} \equiv \mathcal{P} \frac{1}{x}=\frac{\partial}{\partial x} \log |x| .
\end{aligned}
$$


The function $\log |x|$ is a regular function because it is locally integrable at $x=0$ and all canonical regularizations of power functions $1 / x^{n}$ can be defined as derivatives of $\log |x|$ ( Gel'fand et al. [6, 39] omit the regularization symbol $\mathcal{R}$ when discussing the functions $\mathcal{R}\left(1 / x^{n}\right)$ but understand these functions in the sense of canonical regularization ). The canonical regularization of $1 / x^{2}$ can be defined by

$$
\begin{aligned}
& \left(\mathcal{R} \frac{1}{x^{2}}, \varphi(x)\right) \equiv \int_{-\infty}^{+\infty} d x\left(\mathcal{R} \frac{1}{x^{2}}\right) \varphi(x) \equiv \int_{0}^{+\infty} d x \frac{\varphi(x)+\varphi(-x)-2 \varphi(0)}{x^{2}}, \\
& \mathcal{R} \frac{1}{x^{2}}=-\frac{\partial}{\partial x} \mathcal{R} \frac{1}{x}=-\frac{\partial^{2}}{\partial x^{2}} \log |x| .
\end{aligned}
$$

The proof of this formula can be given by the following chain of transformations including partial integration and using (2.14)

$$
\begin{aligned}
\left(\mathcal{R} \frac{1}{x^{2}}, \varphi(x)\right) & =-\left(-\mathcal{R} \frac{1}{x}, \varphi^{(1)}(x)\right) \\
& =\int_{0}^{+\infty} d x \frac{\varphi^{(1)}(x)-\varphi^{(1)}(-x)}{x} \\
& =\int_{0}^{+\infty} d x \frac{1}{x} \frac{\partial}{\partial x}(\varphi(x)+\varphi(-x)-2 \varphi(0)) \\
& =-\int_{0}^{+\infty} d x \frac{\varphi(x)+\varphi(-x)-2 \varphi(0)}{-x^{2}}-\left\{\frac{\varphi(x)+\varphi(-x)-2 \varphi(0)}{x}\right\}_{x=0} .
\end{aligned}
$$

The last expression in braces taken at $x=0$ vanishes and thus Eq.(2.15) is proved. All other proofs of canonical regularizations can be made in analogy to this proof which is here given as an example for such kind of proofs. The meaning of $(\mathcal{P}(1 / x))^{(1)}$ in the inversion formulas for the Radon transform is therefore that we have to substitute it by $-\mathcal{R}\left(1 / x^{2}\right)$ considered in the sense of Eq.(2.15). One may think that this does not give the right sign but it is not so. As an example we consider $\varphi(x)=\exp \left(-x^{2}\right)$

$$
\begin{aligned}
& \left(\mathcal{R} \frac{1}{x^{2}}, \exp \left(-x^{2}\right)\right) \equiv \int_{0}^{+\infty} d x \frac{2\left(\exp \left(-x^{2}\right)-1\right)}{x^{2}} \\
= & \int_{0}^{+\infty} d x \frac{-4 x \exp \left(-x^{2}\right)}{x} \equiv\left(\mathcal{R} \frac{1}{x},-2 x \exp \left(-x^{2}\right)\right)=-2 \sqrt{\pi} .
\end{aligned}
$$

It could seem that $\mathcal{R}\left(1 / x^{2}\right)$ and $\exp \left(-x^{2}\right)$ are both positively definite functions ( $\exp \left(-x^{2}\right)$ is really positively definite ) but, nevertheless, the value of the linear functional in (2.17) for the considered basis function gives a negative number as shown. This means that $\mathcal{R}\left(1 / x^{2}\right)$ is not a positively definite function.

The two-dimensional Radon transformation (2.2) together with its inversion (2.13) is equivalent to the following resolution of the two-dimensional identity operator

$$
-\frac{|c|}{2 \pi^{2}} \int d u \wedge d v\left(\mathcal{R} \frac{1}{(c-u q-v p)^{2}}\right) \delta\left(c-u q^{\prime}-v p^{\prime}\right)=\delta\left(q-q^{\prime}\right) \delta\left(p-p^{\prime}\right) .
$$


All the seeming difficulties with the inverse two-dimensional Radon transform can be avoided if the integration leading to the inversion in two steps via the Fourier transform as the intermediate step is not made at an early stage of the calculations. 


\section{Transformation properties of the Radon transform of the Wigner quasiprobability}

The Wigner quasiprobability can be obtained by forming the trace over the product of the density operator with a transition operator. Among all quasiprobabilities it has the unique property that the operators for the transition from the density operator to the quasiprobability and back from the quasiprobability to the density operator via integration are the same. The transition operators possess many equivalent representations. For our purposes we use the following convenient representation (e.g., 38 )

$$
\begin{aligned}
& W(q, p)=\left\langle\varrho \exp \left(-Q \frac{\partial}{\partial q}-P \frac{\partial}{\partial p}\right)\right\rangle \delta(q) \delta(p), \quad \int d q \wedge d p W(q, p)=1, \\
& \varrho=2 \hbar \pi \int d q \wedge d p W(q, p) \exp \left(-Q \frac{\partial}{\partial q}-P \frac{\partial}{\partial p}\right) \delta(q) \delta(p), \quad\langle A\rangle \equiv \operatorname{Trace}\{A\}
\end{aligned}
$$

where we use the very rational notation $\langle A\rangle$ for the trace of an operator $A$. We now investigate the transformation properties of the Radon transform of the Wigner quasiprobability under displacement and squeezing transformations of the density operator.

Suppose that there is given a density operator $\varrho_{0}$ corresponding to a Wigner quasiprobability $W_{0}(q, p)$. Let us make a displacement of the state described by the density operator $\varrho_{0}$ by using the displacement operator $D(q, p)$ in the following way

$$
\varrho=D(\bar{q}, \bar{p}) \varrho_{0}(D(\bar{q}, \bar{p}))^{\dagger}, \quad D(q, p) \equiv \exp \left\{-\frac{i}{\hbar}(q P-p Q)\right\}=(D(-q,-p))^{\dagger} .
$$

Then we find for the Wigner quasiprobability $W(q, p)$ of the displaced state $\varrho$

$$
\begin{aligned}
W(q, p) & =\left\langle\varrho_{0}(D(\bar{q}, \bar{p}))^{\dagger} \exp \left(-Q \frac{\partial}{\partial q}-P \frac{\partial}{\partial p}\right) D(\bar{q}, \bar{p})\right\rangle \delta(q) \delta(p) \\
& =\left\langle\varrho_{0} \exp \left\{-(Q+\bar{q} I) \frac{\partial}{\partial q}-(P+\bar{p} I) \frac{\partial}{\partial p}\right\}\right\rangle \delta(q) \delta(p)=W_{0}(q-\bar{q}, p-\bar{p})
\end{aligned}
$$

meaning that the new Wigner quasiprobability is simply the displaced old Wigner quasiprobability in the phase plane. This property is also true for all other quasiprobabilities after 
displacement of the state due to the displacement structure of the quasiprobabilities themselves 38]. The Radon transform of the primary and of the transformed state are then connected by a displacement of the variable $c$

$$
\breve{W}(u, v ; c)=\breve{W}_{0}(u, v ; c-u \bar{q}-v \bar{p}),
$$

whereas the Fourier transforms are connected by

$$
\tilde{W}(u, v)=\exp \{-i(u \bar{q}+v \bar{p})\} \tilde{W}_{0}(u, v),
$$

that means by multiplication with a phase factor depending on $(u, v)$ and on the displacement $(\bar{q}, \bar{p})$.

Our next aim is to consider the influence of unitary squeezing transformations of a state described by the density operator onto the Wigner quasiprobability and its Radon and Fourier transforms. We prepare this by considering first some basic formulae for the, in general, nonunitary squeezing operator $S(\xi, \eta, \zeta)$ which is defined by

$$
\begin{aligned}
S(\xi, \eta, \zeta) & \equiv \exp \left\{\frac{\xi}{2} a^{2}+i \frac{\eta}{2}\left(a a^{\dagger}+a^{\dagger} a\right)-\frac{\zeta}{2} a^{\dagger 2}\right\} \\
& =\exp \left\{\frac{i}{2 \hbar}\left(\frac{-i(\xi-\zeta)+2 \eta}{2} Q^{2}+\frac{\xi+\zeta}{2}(Q P+P Q)+\frac{i(\xi-\zeta)+2 \eta}{2} P^{2}\right)\right\} \\
& =\left(S\left(-\zeta^{*},-\eta^{*},-\xi^{*}\right)\right)^{\dagger} .
\end{aligned}
$$

The transformation of the canonical operators $(Q, P)$ by the general squeezing operator is given by ( in representation by the boson operators $\left(a, a^{\dagger}\right)$ this is given in [40] and more specially in 41] )

$$
\begin{aligned}
& S(\xi, \eta, \zeta)(Q, P)(S(\xi, \eta, \zeta))^{-1} \\
= & (Q, P)\left(\begin{array}{cc}
\operatorname{ch} \varepsilon+\frac{\xi+\zeta}{2} \frac{\operatorname{sh} \varepsilon}{\varepsilon}, & \frac{i(\xi-\zeta)-2 \eta}{2} \frac{\operatorname{sh} \varepsilon}{\varepsilon} \\
\frac{i(\xi-\zeta)+2 \eta}{2} \frac{\operatorname{sh} \varepsilon}{\varepsilon}, & \operatorname{ch} \varepsilon-\frac{\xi+\zeta}{2} \frac{\operatorname{sh} \varepsilon}{\varepsilon}
\end{array}\right) \\
\equiv & (Q, P)\left(\begin{array}{cc}
\alpha, & \beta \\
\gamma, & \delta
\end{array}\right), \quad\left|\begin{array}{cc}
\alpha, & \beta \\
\gamma, & \delta
\end{array}\right|=1, \quad \varepsilon \equiv \sqrt{\xi \zeta-\eta^{2}},
\end{aligned}
$$

with the inverse correspondence

$$
\begin{aligned}
& \xi=\frac{\alpha-i \beta-i \gamma-\delta}{2} \frac{\operatorname{Arsh} \vartheta}{\vartheta}, \quad \eta=-\frac{\beta-\gamma}{2} \frac{\operatorname{Arsh} \vartheta}{\vartheta}, \quad \zeta=\frac{\alpha+i \beta+i \gamma-\delta}{2} \frac{\operatorname{Arsh} \vartheta}{\vartheta}, \\
& \vartheta \equiv \sqrt{\left(\frac{\alpha+\delta}{2}\right)^{2}-1}= \pm \operatorname{sh} \varepsilon .
\end{aligned}
$$

This is the fundamental two-dimensional representation of the $S L(2, C) \sim S p(2, C)$-group which in case of unitary squeezing operators $S\left(\zeta^{\prime *}, \eta^{\prime}=\eta^{\prime *}, \zeta^{\prime}\right)$ reduces two the $S L(2, R) \sim$ 
$S p(2, R) \sim S U(1,1)$-group [42, 43]. The matrices of this representation in (3.7) are complex unimodular or symplectic but in general not unitary. Symplectic transformations in spaces of even dimensionality are linear canonical transformations. They preserve an antisymmetric 2-form which can be nondegenerate only in spaces of even dimensionality whereas unimodular transformations preserve a fully antisymmetric $n$-form ( volume form ) in arbitrary $n$-dimensional spaces. Only in two-dimensional spaces one has coincidence between symplectic and unimodular transformations. The notions of symplectic transformations and symplectic groups were introduced by Weyl [44]. We mention here that all problems concerning the multiplication of squeezing operators or the decomposition of squeezing operators into products with certain ordering are solvable by applying the fundamental representation of the two-dimensional symplectic or unimodular group in Eqs.(3.7) and (3.8) (see also [45] and [38]).

In the special case

$$
\xi=\zeta=0, \quad \eta=\eta^{*} \equiv \varphi, \quad S(0, \varphi, 0)=\exp \left(i \frac{\varphi}{2}\right) R(\varphi), \quad R(\varphi) \equiv \exp \left(i \varphi a^{\dagger} a\right),
$$

this operation reduces to rotations according to

$$
R(\varphi)(Q, P)(R(\varphi))^{\dagger}=(Q, P)\left(\begin{array}{cc}
\cos \varphi, & -\sin \varphi \\
\sin \varphi, & \cos \varphi
\end{array}\right)=(Q \cos \varphi+P \sin \varphi,-Q \sin \varphi+P \cos \varphi),
$$

where $R(\varphi)$ denotes the rotation operator.

We now consider the following squeezing transformation of the primary state $\varrho_{0}$ by a unitary squeezing operator $S\left(\zeta^{\prime *}, \eta^{\prime}=\eta^{\prime *}, \zeta^{\prime}\right)$ according to

$$
\varrho=S\left(\zeta^{*}, \eta^{\prime}, \zeta^{\prime}\right) \varrho_{0}\left(S\left(\zeta^{*}, \eta^{\prime}, \zeta^{\prime}\right)\right)^{\dagger}, \quad\left(\eta^{\prime}=\eta^{* *}\right),
$$

where the unitary squeezing operator $S\left(\zeta^{\prime *}, \eta^{\prime}=\eta^{\prime *}, \zeta^{\prime}\right)$ is a special case of the, in general, nonunitary squeezing operator $S(\xi, \eta, \zeta)$ defined in Eq.(3.6). By using the transformation of the basic canonical operators $(Q, P)$ given in (3.7) and taking into account $\left(S\left(\zeta^{\prime *}, \eta^{\prime}=\eta^{\prime *}, \zeta^{\prime}\right)\right)^{\dagger}=S\left(-\zeta^{\prime *},-\eta^{\prime}=-\eta^{\prime *},-\zeta^{\prime}\right)$ one finds

$$
\begin{aligned}
W(q, p) & =\left\langle\varrho_{0}\left(S\left(\zeta^{* *}, \eta^{\prime}, \zeta^{\prime}\right)\right)^{\dagger} \exp \left(-Q \frac{\partial}{\partial q}-P \frac{\partial}{\partial p}\right) S\left(\zeta^{*}, \eta^{\prime}, \zeta^{\prime}\right)\right\rangle \delta(q) \delta(p) \\
& =\left\langle\varrho_{0} \exp \left\{-(Q \delta-P \gamma) \frac{\partial}{\partial q}-(-Q \beta+P \alpha) \frac{\partial}{\partial p}\right\}\right\rangle \delta(q) \delta(p) \\
& =\left\{\left\langle\varrho_{0} \exp \left(-Q \frac{\partial}{\partial q^{\prime}}-P \frac{\partial}{\partial p^{\prime}}\right)\right\rangle \delta\left(q^{\prime}\right) \delta\left(p^{\prime}\right)\right\}_{\left(q^{\prime}=\alpha q+\gamma p, p^{\prime}=\beta q+\delta p\right)} \\
& =W_{0}(\alpha q+\gamma p, \beta q+\delta p) .
\end{aligned}
$$

This means that the Wigner quasiprobability $W(q, p)$ for the state after squeezing can be obtained from the initial Wigner quasiprobability $W_{0}(q, p)$ by an argument transformation 
with a real unimodular or symplectic matrix in the following way

$$
\begin{aligned}
& W(q, p)=W_{0}(\alpha q+\gamma p, \beta q+\delta p) \equiv W_{0}\left(q^{\prime}, p^{\prime}\right), \quad\left(q^{\prime}, p^{\prime}\right)=(q, p)\left(\begin{array}{cc}
\alpha, & \beta \\
\gamma, & \delta
\end{array}\right), \\
& \left(\begin{array}{cc}
\alpha, & \beta \\
\gamma, & \delta
\end{array}\right)=\left(\begin{array}{cc}
\operatorname{ch} \varepsilon^{\prime}+\frac{\zeta^{\prime}+\zeta^{\prime *}}{2} \frac{\operatorname{sh} \varepsilon^{\prime}}{\varepsilon^{\prime}}, & \frac{-i\left(\zeta^{\prime}-\zeta^{\prime *}\right)-2 \eta^{\prime}}{2} \frac{\operatorname{sh} \varepsilon^{\prime}}{\varepsilon^{\prime}} \\
\frac{-i\left(\zeta^{\prime}-\zeta^{\prime *}\right)+2 \eta^{\prime}}{2} \frac{\operatorname{sh} \varepsilon^{\prime}}{\varepsilon^{\prime}}, & \operatorname{ch} \varepsilon^{\prime}-\frac{\zeta^{\prime}+\zeta^{\prime *}}{2} \frac{\operatorname{sh} \varepsilon^{\prime}}{\varepsilon^{\prime}}
\end{array}\right), \\
& \varepsilon^{\prime} \equiv \sqrt{\zeta^{\prime} \zeta^{\prime *}-\eta^{\prime 2}}, \quad\left(\eta^{\prime}=\eta^{*}\right) .
\end{aligned}
$$

This result was originally obtained by Ekert and Knight 46, 47].

The Radon transform $\breve{W}(u, v ; c)$ of the Wigner quasiprobability after squeezing of the state can be obtained from the Radon transform $\breve{W}_{0}(u, v ; c)$ of the Wigner quasiprobability of the initial state by ( the double meaning of the symbol $\delta$ in the following formula can hardly lead to confusion )

$$
\begin{aligned}
\breve{W}(u, v ; c) & =\int d q \wedge d p \delta(c-u q-v p) W(q, p) \\
& =\int d q \wedge d p \delta(c-u q-v p) W_{0}(\alpha q+\gamma p, \beta q+\delta p) \\
& =\int d q^{\prime} \wedge d p^{\prime} \delta\left(c-(\delta u-\beta v) q^{\prime}-(-\gamma u+\alpha v) p^{\prime}\right) W_{0}\left(q^{\prime}, p^{\prime}\right) \\
& =\breve{W}_{0}(\delta u-\beta v,-\gamma u+\alpha v ; c),
\end{aligned}
$$

which can be also written in the form

$$
\begin{aligned}
& \breve{W}(u, v ; c)=\breve{W}_{0}(\delta u-\beta v,-\gamma u+\alpha v ; c) \equiv \breve{W}_{0}\left(u^{\prime}, v^{\prime} ; c\right), \\
& \left(\begin{array}{c}
u^{\prime} \\
v^{\prime}
\end{array}\right)=\left(\begin{array}{rr}
\delta, & -\beta \\
-\gamma, & \alpha
\end{array}\right)\left(\begin{array}{l}
u \\
v
\end{array}\right)=\left(\begin{array}{rr}
\alpha, & \beta \\
\gamma, & \delta
\end{array}\right)^{-1}\left(\begin{array}{l}
u \\
v
\end{array}\right) .
\end{aligned}
$$

The transformation of the variables $(u, v)$ in the Radon transform is made with the contragredient ( inverse transposed ) matrix to the matrix of the transformation of the canonical variables $(q, p)$ if we consider $(u, v)$ as a row vector and by the inverse matrix if we consider $(u, v)$ as a column vector. The same is true for the transformation of the variables $(u, v)$ of the Fourier transform for which one now easily finds

$$
\tilde{W}(u, v)=\tilde{W}_{0}(\delta u-\beta v,-\gamma u+\alpha v) \equiv \tilde{W}_{0}\left(u^{\prime}, v^{\prime}\right) .
$$

It could appear that it is more simple to derive first this result for the Fourier transform of the Wigner quasiprobability and then the corresponding result for the Wigner quasiprobability itself but, really, both derivations are of the same degree of difficulty and very similar.

The combination $u q+v p$ is invariant with respect to the considered unitary squeezing transformations of states

$$
u^{\prime} q^{\prime}+v^{\prime} p^{\prime}=\left(q^{\prime}, p^{\prime}\right)\left(\begin{array}{c}
u^{\prime} \\
v^{\prime}
\end{array}\right)=(q, p)\left(\begin{array}{l}
u \\
v
\end{array}\right)=u q+v p
$$


but not with respect to displacements of states. One can make both displacement and squeezing transformations of the initial state but then the result for the transformed Wigner quasiprobability and its Radon and Fourier transforms depends on the order of these operations. If we make first the squeezing operation and after this the displacement then we can immediately apply the derived transformation properties. However, if we make first the displacement and after this the squeezing of the state then we have to take into account that the displacement parameters must be transformed by the real unimodular or symplectic matrices in the same way as the corresponding coordinates. We do not explicitly write this down. Usually, the Radon transform $\breve{W}(u, v ; c)$ of the Wigner quasiprobability $W(q, p)$ is only considered in the special form $W\left(\cos \varphi, \sin \varphi ; q_{\varphi}\right)$ and denoted by $w\left(\varphi, q_{\varphi}\right)$ but it is difficult to express such a fundamental transformation property as in Eq.(3.15) in the last notation. Let us also mention that the transformation properties of all other quasiprobabilities and their Radon and Fourier transforms with respect to the considered squeezing transformations of states are not so simple as for the Wigner quasiprobability and we will not consider this here.

Mancini, Man'ko and Tombesi [28, 29] consider practically the same object $\breve{W}(u, v ; c)$ which is the Radon transform of the Wigner quasiprobability ( slightly extended by a further displacement ) and call this symplectic tomography. Since $\breve{W}(u, v ; c)$ contains the complete information about the state one can transform the equations of time evolution for the state from any other quantity with complete information, for example, for the density operator or for the Wigner quasiprobability to an equation for the Radon transform. A certain advantage of this approach is that we consider then directly the measurable quantities which are positively definite ( or semidefinite ) and can be considered for every fixed $(u, v$ ) as genuine probability densities 28, 29. The noncommutativity of the basic observables in quantum mechanics appears then as the, in general, noncompatibility of all these probability densities for essentially different $(u, v)$ on a classical level that means as marginals or Radon transforms of purely classical distribution functions $f(q, p)$.

Let us shortly consider the transformation of the Wigner quasiprobability in the complex representation with respect to unitary squeezing transformations. The corresponding formula to $(3.7)$ is then

$$
\begin{aligned}
& S(\xi, \eta, \zeta)\left(a, a^{\dagger}\right)(S(\xi, \eta, \zeta))^{-1} \\
= & \left(a, a^{\dagger}\right)\left(\begin{array}{cc}
\operatorname{ch} \varepsilon-i \eta \frac{\operatorname{sh} \varepsilon}{\varepsilon}, & \xi \frac{\operatorname{sh} \varepsilon}{\varepsilon} \\
\zeta \frac{\operatorname{sh} \varepsilon}{\varepsilon}, & \operatorname{ch} \varepsilon+i \eta \frac{\operatorname{sh} \varepsilon}{\varepsilon}
\end{array}\right) \\
\equiv & \left(a, a^{\dagger}\right)\left(\begin{array}{cc}
\kappa, & \lambda \\
\mu, & \nu
\end{array}\right), \quad\left|\begin{array}{cc}
\kappa, & \lambda \\
\mu, & \nu
\end{array}\right|=1, \quad \varepsilon \equiv \sqrt{\xi \zeta-\eta^{2}},
\end{aligned}
$$

with the correspondences

$$
\left(\begin{array}{cc}
\kappa, & \lambda \\
\mu, & \nu
\end{array}\right)=\left(\begin{array}{ll}
\frac{\alpha+i \beta-i \gamma+\delta}{2}, & \frac{\alpha-i \beta-i \gamma-\delta}{2} \\
\frac{\alpha+i \beta+i \gamma-\delta}{2}, & \frac{\alpha-i \beta+i \gamma+\delta}{2}
\end{array}\right), \quad \kappa+\nu=\alpha+\delta
$$




$$
\left(\begin{array}{cc}
\alpha, & \beta \\
\gamma, & \delta
\end{array}\right)=\left(\begin{array}{cc}
\frac{\kappa+\lambda+\mu+\nu}{2}, & -i \frac{\kappa-\lambda+\mu-\nu}{2} \\
i \frac{\kappa+\lambda-\mu-\nu}{2}, & \frac{\kappa-\lambda-\mu+\nu}{2}
\end{array}\right)
$$

Then one finds the transformed Wigner quasiprobability after the unitary squeezing transformation of the state according to (3.11) in analogy to (3.12)

$$
W\left(\alpha, \alpha^{*}\right)=W_{0}\left(\nu^{*} \alpha+\mu \alpha^{*}, \mu^{*} \alpha+\nu \alpha^{*}\right) \equiv W_{0}\left(\alpha^{\prime}, \alpha^{\prime *}\right),
$$

with the following specified unimodular or symplectic matrix due to the unitarity of the considered state transformation

$$
\left(\begin{array}{cc}
\nu^{*}, & \mu^{*} \\
\mu, & \nu
\end{array}\right)=\left(\begin{array}{cc}
\operatorname{ch} \varepsilon^{\prime}-i \eta^{\prime} \frac{\operatorname{sh} \varepsilon^{\prime}}{\varepsilon^{\prime}}, & \zeta^{*} \frac{\operatorname{sh} \varepsilon^{\prime}}{\varepsilon^{\prime}} \\
\zeta^{\prime} \frac{\operatorname{sh} \varepsilon^{\prime}}{\varepsilon^{\prime}}, & \operatorname{ch} \varepsilon^{\prime}+i \eta^{\prime} \frac{\operatorname{sh} \varepsilon^{\prime}}{\varepsilon^{\prime}}
\end{array}\right), \quad \varepsilon^{\prime} \equiv \sqrt{\zeta^{\prime} \zeta^{\prime *}-\eta^{\prime 2}}, \quad\left(\eta^{\prime}=\eta^{\prime *}\right) .
$$

The propagation of the transformation properties (3.20) of the Wigner quasiprobability in complex representation to its Radon and Fourier transforms in complex representation is easily to find if one introduces a certain convention for the definition of these complex representations. We will not do this here.

Let us now consider the more special unitary squeezing operator $S\left(\zeta^{\prime *}, 0, \zeta^{\prime}\right)$ as it is mostly used in the theory of squeezing [48-58]. According to (3.6) we have

$$
\begin{aligned}
S\left(\zeta^{\prime *}, 0, \zeta^{\prime}\right) & =\exp \left\{\frac{\zeta^{*}}{2} a^{2}-\frac{\zeta^{\prime}}{2} a^{\dagger 2}\right\} \\
& =\exp \left(-\frac{\zeta^{\prime}}{2} \frac{\operatorname{th}\left|\zeta^{\prime}\right|}{\left|\zeta^{\prime}\right|} a^{\dagger 2}\right)\left(\operatorname{ch}\left|\zeta^{\prime}\right|\right)^{-\left(N+\frac{1}{2} I\right)} \exp \left(\frac{\zeta^{\prime *}}{2} \frac{\operatorname{th}\left|\zeta^{\prime}\right|}{\left|\zeta^{\prime}\right|} a^{2}\right) \\
& \equiv \exp \left(-\frac{\zeta}{2} a^{\dagger 2}\right)\left(\sqrt{1-\mid \zeta^{2}}\right)^{N+\frac{1}{2} I} \exp \left(\frac{\zeta^{*}}{2} a^{2}\right), \quad N \equiv a^{\dagger} a
\end{aligned}
$$

where there is additionally given an almost normally ordered decomposition into factors ( the normal ordering of the central factor is mostly not necessary in applications but could be easily made ). Furthermore, it is made a new parametrization of the squeezing by the complex variable $\zeta$ characterized by

$$
\zeta=\zeta^{\prime} \frac{\operatorname{th}\left|\zeta^{\prime}\right|}{\left|\zeta^{\prime}\right|}, \quad \zeta^{\prime}=\zeta \frac{\operatorname{Arth}|\zeta|}{|\zeta|}, \quad|\zeta|=\operatorname{th}\left|\zeta^{\prime}\right|, \quad\left|\zeta^{\prime}\right|=\operatorname{Arth}|\zeta| .
$$

The complex parameter $\zeta$ describes the squeezing operation in the nonunitary approach 41, 59] For convenience of applications of the preceding formulae we give here the explicit form of the corresponding specified matrices

$$
\left(\begin{array}{cc}
\alpha, & \beta \\
\gamma, & \delta
\end{array}\right)=\left(\begin{array}{cc}
\operatorname{ch}\left|\zeta^{\prime}\right|+\frac{\zeta^{\prime}+\zeta^{* *}}{2} \frac{\operatorname{sh}\left|\zeta^{\prime}\right|}{\left|\zeta^{\prime}\right|}, & -i \frac{\zeta^{\prime}-\zeta^{* *}}{2} \frac{\operatorname{sh}\left|\zeta^{\prime}\right|}{\left|\zeta^{\prime}\right|} \\
-i \frac{\zeta^{\prime}-\zeta^{\prime *}}{2} \frac{\operatorname{sh}\left|\zeta^{\prime}\right|}{\left|\zeta^{\prime}\right|}, & \operatorname{ch}\left|\zeta^{\prime}\right|-\frac{\zeta^{\prime}+\zeta^{\prime *}}{2} \frac{\operatorname{sh}\left|\zeta^{\prime}\right|}{\left|\zeta^{\prime}\right|}
\end{array}\right)
$$




$$
\begin{aligned}
& =\frac{1}{\sqrt{1-\mid \zeta^{2}}}\left(\begin{array}{cc}
1+\frac{\zeta+\zeta^{*}}{2}, & -i \frac{\zeta-\zeta^{*}}{2} \\
-i \frac{\zeta-\zeta^{*}}{2}, & 1-\frac{\zeta+\zeta^{*}}{2}
\end{array}\right), \\
\left(\begin{array}{cc}
\nu^{*}, & \mu^{*} \\
\mu, & \nu
\end{array}\right) & =\left(\begin{array}{cc}
\operatorname{ch}\left|\zeta^{\prime}\right|, & \zeta^{\prime *} \frac{\operatorname{sh}\left|\zeta^{\prime}\right|}{\left|\zeta^{\prime}\right|} \\
\zeta^{\prime} \frac{\operatorname{sh}\left|\zeta^{\prime}\right|}{\left|\zeta^{\prime}\right|} & \operatorname{ch|\zeta ^{\prime }|}
\end{array}\right)=\frac{1}{\sqrt{1-|\zeta|^{2}}}\left(\begin{array}{cc}
1, & \zeta^{*} \\
\zeta, & 1
\end{array}\right) .
\end{aligned}
$$

Now we can apply all derived transformation formulae for the Wigner quasiprobability and its Radon and Fourier transforms and have to specify the corresponding matrix elements by the expressions given here.

The substitution of the complex squeezing parameter $\zeta^{\prime}$ by $\zeta$ according to Eq.(3.22) is not only a formal substitution but has a deep physical content. If one acts with squeezing and displacement operators onto pure states $|\psi\rangle$ one can represent $|\psi\rangle$ by its Fock-state representation. Then one can represent the Fock states by the action of powers of the creation operator onto the vacuum state $|0\rangle$ that means by an excitation operator. Then one can bring the squeezing operator of the form (3.22) by application of the commutation rules with the excitation operator in the position that it acts in unchanged form directly onto the vacuum state. By using the ordered decomposition given in (3.22) the operator $\exp \left(\left(\zeta^{*} / 2\right) a^{2}\right)$ acts onto the vacuum state as the unity operator whereas $\left(\sqrt{1-|\zeta|^{2}}\right)^{N+(1 / 2) I}$ multiplies it with a normalization factor $\left(1-|\zeta|^{2}\right)^{1 / 4}$. The essential action of the squeezing operator onto the vacuum state can be then substituted by the action of the nonunitary squeezing operator $\exp \left(-(\zeta / 2) a^{\dagger 2}\right)$. After this the changed excitation and displacement operators act onto the obtained state that must be considered for the concrete case. This procedure should be also possible for mixed states or genuine density operators. Hence the squeezing parameter $\zeta$ in the nonunitary approch possesses a concrete physical meaning. In case of the more general unitary squeezing operator $S\left(\zeta^{* *}, \eta^{\prime}=\eta^{*}, \zeta^{\prime}\right)$ one has 1 complex parameter $\zeta^{\prime}$ and 1 real parameter $\eta^{\prime}$ that is more than the only 1 complex parameter $\zeta$ in the nonunitary approach and there remains a certain freedom in the choice of the correspondence between unitary and nonunitary approaches [40. We do not discuss this here.

As an example we consider the vacuum state $\varrho_{0}=|0\rangle\langle 0|$. The Wigner quasiprobability and its Radon and Fourier transforms are

$$
\begin{aligned}
& W_{0}(q, p)=\frac{1}{\hbar \pi} \exp \left\{-\frac{q^{2}+p^{2}}{\hbar}\right\}, \\
& \breve{W}_{0}(u, v ; c)=\frac{1}{\sqrt{\hbar \pi\left(u^{2}+v^{2}\right)}} \exp \left\{-\frac{c^{2}}{\hbar\left(u^{2}+v^{2}\right)}\right\}, \\
& \tilde{W}_{0}(u, v)=\exp \left\{-\frac{\hbar\left(u^{2}+v^{2}\right)}{4}\right\} .
\end{aligned}
$$

After squeezing of the state with the special unitary squeezing operator in Eq.(3.23) and succeeding displacement one obtains squeezed coherent states and by using Eqs.(3.3)-(3.5) 
and (3.13)-(3.16) as well as (3.24) one finds the following Wigner quasiprobability and its Radon and Fourier transforms [25]

$$
\begin{aligned}
W(q, p)= & \frac{1}{\hbar \pi} \exp \left\{-\frac{|1+\zeta|^{2}(q-\bar{q})^{2}+|1-\zeta|^{2}(p-\bar{p})^{2}-i\left(\zeta-\zeta^{*}\right) 2(q-\bar{q})(p-\bar{p})}{\hbar\left(1-|\zeta|^{2}\right)}\right\}, \\
\breve{W}(u, v ; c)= & \sqrt{\frac{1-|\zeta|^{2}}{\hbar \pi\left(|1-\zeta|^{2} u^{2}+|1+\zeta|^{2} v^{2}+i\left(\zeta-\zeta^{*}\right) 2 u v\right)}} \\
& \times \exp \left\{-\frac{\left(1-|\zeta|^{2}\right)(c-u \bar{q}-v \bar{p})^{2}}{\hbar\left(|1-\zeta|^{2} u^{2}+|1+\zeta|^{2} v^{2}+i\left(\zeta-\zeta^{*}\right) 2 u v\right)}\right\}, \\
\tilde{W}(u, v)= & \exp \{-i(u \bar{q}+v \bar{p})\} \\
& \times \exp \left\{-\frac{\hbar\left(|1-\zeta|^{2} u^{2}+|1+\zeta|^{2} v^{2}+i\left(\zeta-\zeta^{*}\right) 2 u v\right)}{4\left(1-|\zeta|^{2}\right)}\right\} .
\end{aligned}
$$

We mention the following complex factorizations of the numerators and denominators in the exponents which are possible with rational coefficients in $\zeta$ and $\zeta^{*}$ only for the Wigner quasiprobability and its Radon and Fourier transforms but not for other important quasiprobabilities of these states such as, for example, the coherent-state quasiprobability.

$$
\begin{aligned}
& |1+\zeta|^{2} q^{2}+|1-\zeta|^{2} p^{2}-i\left(\zeta-\zeta^{*}\right) 2 q p=\{(1+\zeta) q+i(1-\zeta) p\}\left\{\left(1+\zeta^{*}\right) q-i\left(1-\zeta^{*}\right) p\right\}, \\
& |1-\zeta|^{2} u^{2}+|1+\zeta|^{2} v^{2}+i\left(\zeta-\zeta^{*}\right) 2 u v=\{(1-\zeta) u+i(1+\zeta) v\}\left\{\left(1-\zeta^{*}\right) u-i\left(1+\zeta^{*}\right) v\right\} .
\end{aligned}
$$

They express a certain duality of the transformation of the variables in the Wigner quasiprobability on one side and its Radon and Fourier transform on the other side.

For convenience we give in the following formula the connection between our parameters $\bar{q}, \bar{p}$ and $\zeta$ for squeezed coherent states and the expectation values of the canonical operators $(Q, P)$ and their dispersions and symmetrical correlation $(\bar{A} \equiv\langle\varrho A\rangle, \Delta Q \equiv Q-\bar{Q} I, \Delta P \equiv$ $P-\bar{P} I)$

$$
\begin{aligned}
& \bar{Q}=\bar{q}, \quad \bar{P}=\bar{p}, \quad \overline{(\Delta Q)^{2}}=\frac{\hbar}{2} \frac{|1-\zeta|^{2}}{1-|\zeta|^{2}}, \quad \overline{(\Delta P)^{2}}=\frac{\hbar}{2} \frac{|1+\zeta|^{2}}{1-|\zeta|^{2}} \\
& \frac{1}{2}(\overline{\Delta Q \Delta P}+\overline{\Delta P \Delta Q})=i \frac{\hbar}{2} \frac{\zeta-\zeta^{*}}{1-|\zeta|^{2}}
\end{aligned}
$$

These 5 real parameters can be used for the unique characterization of squeezed coherent states. Because of

$$
\overline{(\Delta Q(\varphi))^{2}} \overline{(\Delta P(\varphi))^{2}}-\frac{1}{4}(\overline{\Delta Q(\varphi) \Delta P(\varphi)}+\overline{\Delta P(\varphi) \Delta Q(\varphi)})^{2}=\frac{\hbar^{2}}{4},
$$

for arbitrary $\varphi$ they effectively reduce to 4 independent parameters for squeezed coherent states. However, for general Gaussian Wigner quasiprobabilities corresponding to displaced and squeezed thermal states these parameters become independent and (3.39) has to be 
substituted by an inequality corresponding to a modification of the usual uncertainty relations by inclusion of the uncertainty correlation [60-62]. The maximal and minimal values of $\sqrt{\overline{(\Delta Q(\varphi))^{2}}}$ for varying $\varphi$ are determined by

$$
\begin{aligned}
& \sqrt{\overline{\left(\Delta Q\left(\varphi_{\max }\right)\right)^{2}}}=\sqrt{\frac{\hbar}{2} \frac{1+|\zeta|}{1-|\zeta|}}, \quad \sqrt{\overline{\left(\Delta Q\left(\varphi_{\min }\right)\right)^{2}}}=\sqrt{\frac{\hbar}{2} \frac{1-|\zeta|}{1+|\zeta|}} \\
& \exp \left(i 2 \varphi_{\max }\right)=-\frac{\zeta}{|\zeta|}, \quad \exp \left(i 2 \varphi_{\min }\right)=+\frac{\zeta}{|\zeta|}, \longrightarrow \exp \left(i 4 \varphi_{\text {ext }}\right)=\frac{\zeta}{\zeta^{*}}
\end{aligned}
$$

These uncertainties can be considered as one of the possible definitions of the widths of the principal axes of the squeezing ellipse. For the corresponding angles $\varphi_{\text {ext }}$ determined by (3.30) the symmetrical correlation vanishes, i.e.

$$
\overline{\Delta Q\left(\varphi_{\text {ext }}\right) \Delta P\left(\varphi_{\text {ext }}\right)}+\overline{\Delta P\left(\varphi_{\text {ext }}\right) \Delta Q\left(\varphi_{\text {ext }}\right)}=0
$$

In an analogous manner one can consider squeezing and displacement of other initial states, for example, Fock states, thermal states or Schrödinger cat states. 


\section{Reconstruction of the density operator from the Radon transform}

In this section we consider the reconstruction of the density operator and of its matrix elements in the Fock-state basis from the Radon transform of the Wigner quasiprobability. Let us first establish the following relation between the Radon transform and the rotated marginals of the Wigner quasiprobability

$$
\breve{W}(\cos \varphi, \sin \varphi ; q)=\left\langle q\left|(R(\varphi))^{\dagger} \varrho R(\varphi)\right| q\right\rangle \equiv\langle q ; \varphi|\varrho| q ; \varphi\rangle
$$

where $|q ; \varphi\rangle$ denotes the orthonormalized eigenstates of the rotated canonical operator $Q(\varphi)$ to eigenvalues $q$ in the following way ( see Eq.(3.10))

$$
\begin{aligned}
& Q(\varphi)|q ; \varphi\rangle=R(\varphi) Q(R(\varphi))^{\dagger} R(\varphi)|q\rangle=q|q ; \varphi\rangle, \quad Q|q\rangle=q|q\rangle, \quad|q ; \varphi\rangle \equiv R(\varphi)|q\rangle, \\
& \left\langle q ; \varphi \mid q^{\prime} ; \varphi\right\rangle=\delta\left(q-q^{\prime}\right), \quad \int_{-\infty}^{+\infty} d q|q ; \varphi\rangle\langle q ; \varphi|=I .
\end{aligned}
$$

To prove Eq.(4.1) we first immediately find the special result

$$
\langle q|\varrho| q\rangle=\int_{-\infty}^{+\infty} d p W(q, p)=\breve{W}(1,0 ; q) .
$$

Then, we see from Eq.(4.1) that $\langle q ; \varphi|\varrho| q ; \varphi\rangle$ corresponds to the Wigner quasiprobability of a rotated density operator about an angle $-\varphi$ that means to $R(-\varphi) \varrho(R(-\varphi))^{\dagger}$ and according to Eqs.(3.10), (3.12), (3.14) or the definition of the Radon transform in Eq.(2.2) one obtains

$$
\langle q ; \varphi|\varrho| q ; \varphi\rangle=\int_{-\infty}^{+\infty} d p W(q \cos \varphi-p \sin \varphi, q \sin \varphi+p \cos \varphi)=\breve{W}(\cos \varphi, \sin \varphi ; q) .
$$

Thus the relation (4.1) is proved. From the well-known position representation $\langle q \mid n\rangle$ of the Fock states $|n\rangle$ it follows

$$
\langle q ; \varphi \mid n\rangle=\langle q|R(-\varphi)| n\rangle=\frac{\exp (-i n \varphi)}{(\hbar \pi)^{\frac{1}{4}}} \exp \left(-\frac{q^{2}}{2 \hbar}\right) \frac{1}{\sqrt{2^{n} n !}} H_{n}\left(\frac{q}{\sqrt{\hbar}}\right) .
$$

With the summation formula of Mehler 63

$$
\sum_{n=0}^{\infty} \frac{z^{n}}{2^{n} n !} H_{n}(x) H_{n}(y)=\frac{1}{\sqrt{1-z^{2}}} \exp \left\{\frac{2 x y z-\left(x^{2}+y^{2}\right) z^{2}}{1-z^{2}}\right\},
$$


which is not difficult to prove [40] one calculates by Fock-state expansion the more general scalar product ( Green's function to the wave equation for the harmonic oscillator )

$$
\left\langle q ; \varphi \mid q^{\prime} ; \varphi^{\prime}\right\rangle=\frac{1}{\sqrt{\hbar \pi\left(1-\exp \left\{-i 2\left(\varphi-\varphi^{\prime}\right)\right\}\right)}} \exp \left\{i \frac{\left(q^{2}+q^{2}\right) \cos \left(\varphi-\varphi^{\prime}\right)-2 q q^{\prime}}{2 \hbar \sin \left(\varphi-\varphi^{\prime}\right)}\right\} .
$$

In the limiting case $\varphi^{\prime} \rightarrow \varphi$ one obtains the delta function $\delta\left(q-q^{\prime}\right)$ and in case of $\varphi^{\prime}=\varphi+\pi / 2$ the scalar product $\langle q \mid p\rangle$ if one substitutes $q^{\prime} \equiv p$.

The Wigner quasiprobability contains the complete information about the state described by the density operator. The reconstruction of the density operator from the Wigner quasiprobability can be made by transition operators. As was already mentioned the Wigner quasiprobability has the unique property among all quasiprobabilities that the transition operators for the transition from the density operator to the quasiprobability by forming the trace and from the quasiprobability back to the density operator by a phase-space integral are the same. The reconstruction formula of the density operator from the Wigner quasiprobability by means of these transition operators $T_{0}(q, p)$ in real or $T_{0}\left(\alpha, \alpha^{*}\right)$ in complex representation has the following form

$$
\begin{aligned}
& \varrho=2 \hbar \pi \int d q \wedge d p W(q, p) T_{0}(q, p)=\pi \int \frac{i}{2} d \alpha \wedge d \alpha^{*} W\left(\alpha, \alpha^{*}\right) T_{0}\left(\alpha, \alpha^{*}\right), \\
& W(q, p)=\left\langle\varrho T_{0}(q, p)\right\rangle, \quad W\left(\alpha, \alpha^{*}\right)=\left\langle\varrho T_{0}\left(\alpha, \alpha^{*}\right)\right\rangle .
\end{aligned}
$$

The transition operator for the Wigner quasiprobability is essentially the displaced parity operator [64-66,38]. There exist many representations for this transition operator. We use for our purpose the representation explicitly contained in Eq.(3.1). The reconstruction formula (4.8) can be written then in real representation in the following form

$$
\begin{aligned}
\varrho & =2 \hbar \pi \int d q \wedge d p W(q, p) \exp \left(-Q \frac{\partial}{\partial q}-P \frac{\partial}{\partial p}\right) \delta(q) \delta(p) \\
& =2 \hbar \pi \int d q \wedge d p \delta(q) \delta(p) \exp \left(Q \frac{\partial}{\partial q}+P \frac{\partial}{\partial p}\right) W(q, p) \\
& =2 \hbar \pi \sum_{k=0}^{\infty} \sum_{l=0}^{\infty} \frac{\mathcal{S}\left\{Q^{k} P^{l}\right\}}{k ! l !}\left\{\frac{\partial^{k+l}}{\partial q^{k} \partial p^{l}} W(q, p)\right\}_{(q=0, p=0)} .
\end{aligned}
$$

where $\mathcal{S}\{\ldots\}$ means symmetrical ordering of the content in braces. This formula shows first of all that the Wigner quasiprobability as all other quasiprobabilities too contains a great redundancy and that for the reconstruction of the density operator it is already sufficient to know the Wigner quasiprobability $W(q, p)$ in an arbitrarily small neighbourhood of $(q=0, p=0)$. One could introduce in Eq.(4.9) the representation of the Wigner quasiprobability $W(q, p)$ by its Radon transform $\breve{W}(u, v ; q)$ explicitly given in Eq.(2.13) and has a representation of the density operator by the rotated quadrature components. Such a form, however, is not very convenient, for example, for the calculation of the matrix elements of the density operator in the Fock-state representation. It is better for some purposes to use the Fourier transform $\tilde{W}(u, v)$ of the Wigner quasiprobability as an intermediate step. 
The introduction of the Fourier transform of the Wigner quasiprobability according to Eq.(2.7) into Eq.(4.9) yields

$$
\begin{aligned}
\varrho & =2 \hbar \pi \int d q \wedge d p \delta(q) \delta(p) \exp \left(Q \frac{\partial}{\partial q}+P \frac{\partial}{\partial p}\right) \frac{1}{(2 \pi)^{2}} \int d u \wedge d v \tilde{W}(u, v) \exp \{i(u q+v p)\} \\
& =\frac{\hbar}{2 \pi} \int d u \wedge d v \exp \{i(Q u+P v)\} \tilde{W}(u, v) .
\end{aligned}
$$

Now, by expressing the Fourier transform of the Wigner quasiprobability by the Radon transform of the Wigner quasiprobability one finds ( $I$ is the identity operator )

$$
\varrho=\frac{\hbar}{2 \pi} \int_{-\infty}^{+\infty} d c \int d u \wedge d v \exp \{-i(I c-Q u-P v)\} \breve{W}(u, v ; c) .
$$

After the substitutions

$$
u \equiv r \cos \varphi \quad v \equiv r \sin \varphi, \quad c \equiv r q,
$$

one arrives at ( note the general identity $\int_{0}^{+\infty} d r r \int_{0}^{2 \pi} f(r, \varphi)=\int_{-\infty}^{+\infty} d r|r| \int_{0}^{\pi} f(r, \varphi)$ for unique functions of $r$ and $\varphi$ over the plane )

$$
\begin{aligned}
\varrho & =\frac{\hbar}{2 \pi} \int_{0}^{\pi} d \varphi \int_{-\infty}^{+\infty} d q \int_{-\infty}^{+\infty} d r|r| \exp \{-i r(I q-Q \cos \varphi-P \sin \varphi)\} \breve{W}(\cos \varphi, \sin \varphi ; q) \\
& =\frac{1}{\pi} \int_{0}^{\pi} d \varphi \int_{-\infty}^{+\infty} d q \breve{W}(\cos \varphi, \sin \varphi ; q) \hbar \frac{\partial}{\partial q}\left(\mathcal{P} \frac{1}{I q-Q(\varphi)}\right) \\
& =-\frac{1}{\pi} \int_{0}^{\pi} d \varphi \int_{-\infty}^{+\infty} d q\left(\mathcal{P} \frac{1}{I q-Q(\varphi)}\right) \hbar \frac{\partial}{\partial q} \breve{W}(\cos \varphi, \sin \varphi ; q),
\end{aligned}
$$

where $Q(\varphi)$ is the rotated operator $Q$ according to Eq.(3.10). Recall that $\mathcal{P}(1 / x) \equiv \mathcal{R}(1 / x)$ means the principal value of $1 / x$ and its first derivative $-\mathcal{R}\left(1 / x^{2}\right)$ the canonical regularization of $-1 / x^{2}$ as explained in section 2, Eq.(2.15). However, the operator character of the singularities in this formula makes it more difficult to interpret them. A possible way to do this is to make a Taylor series expansion in powers of $Q(\varphi)$ in the following way

$$
\begin{aligned}
\varrho= & -\frac{1}{\pi} \int_{0}^{\pi} d \varphi \int_{-\infty}^{+\infty} d q \hbar \breve{W}(\cos \varphi, \sin \varphi ; q) \mathcal{R} \frac{1}{(I q-Q(\varphi))^{2}} \\
= & -\sum_{k=0}^{\infty}(k+1) \frac{1}{\pi} \int_{0}^{\pi} d \varphi(Q(\varphi))^{k} \int_{-\infty}^{+\infty} d q \hbar \breve{W}(\cos \varphi, \sin \varphi ; q) \mathcal{R} \frac{1}{q^{2+k}} \\
= & -\sum_{k=0}^{\infty}(k+1) \frac{1}{\pi} \int_{0}^{\pi} d \varphi(Q(\varphi))^{k} \\
& \times \int_{0}^{+\infty} d q \frac{\hbar}{q^{2+k}}\left\{\breve{W}(\cos \varphi, \sin \varphi ; q)+(-1)^{k} \breve{W}(\cos \varphi, \sin \varphi ;-q)\right. \\
& \left.-\sum_{l=0}^{k}\left(1+(-1)^{k+l}\right) \frac{q^{l}}{l !} \frac{\partial^{l} \breve{W}}{\partial q^{l}}(\cos \varphi, \sin \varphi ; 0)\right\}
\end{aligned}
$$


In the last part of this representation we have explicitly written down the meaning of the canonical regularization of powers $1 / q^{2+k}$ ( see also [39] ). The integration over $q$ goes here only from zero to plus infinity. We wrote this equation in detail because the content of formulae such as (4.13) was not represented with clarity in the literature about quantum tomography. However, it seems that Eqs.(4.14) does not provide a very convenient approach and mostly it is better to make the integration with respect to $r$ in Eq.(4.13) not before calculating the matrix elements of the density operator in Fock-state representation or in other representation. Nevertheless, it cannot be excluded that sometimes some initial terms of the Taylor series expansion in (4.14) could give already a good approximation that must be investigated but the same approximations can be obviously obtained by Taylor series expansion of the exponentials in (4.13) where the integration over $r$ is accomplished in a later stage of the calculations.

A more convenient form of the reconstruction formula of the density operator from its Radon transform for many purposes can be obtained when the integration with respect to $r$ is not accomplished before making the transition to normal ordering. Our method is similar to the method used in [14, 67]. In such a way by transition to normal ordering one obtains

$$
\begin{aligned}
\varrho= & \frac{1}{\pi} \int_{0}^{\pi} d \varphi \int_{-\infty}^{+\infty} d q \breve{W}(\cos \varphi, \sin \varphi ; q) \frac{\partial}{\partial q}\left(i \frac{\hbar}{2} \int_{-\infty}^{+\infty} d r \frac{r}{|r|} \exp (-i r(\operatorname{Iq}-Q(\varphi)))\right. \\
= & \frac{1}{\pi} \int_{0}^{\pi} d \varphi \int_{-\infty}^{+\infty} d q \breve{W}(\cos \varphi, \sin \varphi ; q) \\
& \times \frac{\partial}{\partial q}\left\{R(\varphi) i \frac{\hbar}{2} \int_{-\infty}^{+\infty} d r \frac{r}{|r|} \exp \left(-i r q-r^{2} \frac{\hbar}{4}\right) \exp \left(i r \sqrt{\frac{\hbar}{2}} a^{\dagger}\right) \exp \left(i r \sqrt{\frac{\hbar}{2}} a\right)(R(\varphi))^{\dagger}\right\},
\end{aligned}
$$

and by the substitution of $r \rightarrow i \partial / \partial q$ in parts of the integral over $r$

$$
\begin{aligned}
\varrho= & \frac{1}{\pi} \int_{0}^{\pi} d \varphi \int_{-\infty}^{+\infty} d q \breve{W}(\cos \varphi, \sin \varphi ; q) \\
& \times \sqrt{\hbar} \frac{\partial}{\partial q}\left\{R(\varphi) \exp \left(-a^{\dagger} \sqrt{\frac{\hbar}{2}} \frac{\partial}{\partial q}\right) \exp \left(-a \sqrt{\frac{\hbar}{2}} \frac{\partial}{\partial q}\right)(R(\varphi))^{\dagger}\right. \\
& \left.\times i \frac{\sqrt{\hbar}}{2} \int_{-\infty}^{+\infty} d r \frac{r}{|r|} \exp \left(-i q r-\frac{\hbar}{4} r^{2}\right)\right\} .
\end{aligned}
$$

We obtained a representation where a normally ordered operator part is separated from an integral onto which it acts. Now, we make the variable substitution

$$
x \equiv \frac{q}{\sqrt{\hbar}}, \quad \frac{\partial}{\partial x}=\sqrt{\hbar} \frac{\partial}{\partial q}, \quad d x=\frac{d q}{\sqrt{\hbar}} .
$$

The integral over $r$ in Eq.(4.16) can be transformed and solved in the following way

$$
\begin{aligned}
& i \frac{\sqrt{\hbar}}{2} \int_{-\infty}^{+\infty} d r \frac{r}{|r|} \exp \left(-i \sqrt{\hbar} x r-\frac{\hbar}{4} r^{2}\right)=2 \exp \left(-x^{2}\right) \int_{0}^{x} d t \exp \left(t^{2}\right) \equiv 2 F(x) \\
= & \sqrt{2} D_{0}(\sqrt{2} x) \frac{i}{2}\left\{D_{-1}(i \sqrt{2} x)-D_{-1}(-i \sqrt{2} x)\right\} \equiv h_{0}(x) g_{0}(x),
\end{aligned}
$$


with the abbreviations

$$
\begin{aligned}
& h_{0}(x) \equiv \frac{1}{\pi^{\frac{1}{4}}} \exp \left(-\frac{x^{2}}{2}\right)=\frac{1}{\pi^{\frac{1}{4}}} D_{0}(\sqrt{2} x), \quad \int_{-\infty}^{+\infty} d x\left(h_{0}(x)\right)^{2}=1, \\
& g_{0}(x) \equiv 2 \pi^{\frac{1}{4}} \exp \left(-\frac{x^{2}}{2}\right) \int_{0}^{x} d t \exp \left(t^{2}\right)=\sqrt{2} \pi^{\frac{1}{4}} \frac{i}{2}\left\{D_{-1}(i \sqrt{2} x)-D_{-1}(-i \sqrt{2} x)\right\} \\
& W\left(h_{0}(x), g_{0}(x)\right)=2, \quad W(f(x), g(x)) \equiv f(x) g^{(1)}(x)-f^{(1)}(x) g(x),
\end{aligned}
$$

where $W(f(x), g(x))$ denotes the Wronskian of two functions $f(x)$ and $g(x)$ and $D_{\nu}(z)$ the functions of the parabolic cylinder, in particular

$$
D_{-1}( \pm i \sqrt{2} x)=\sqrt{\frac{\pi}{2}} \exp \left(-\frac{x^{2}}{2}\right)(1 \mp \Phi(i x)), \quad \Phi(\mu x) \equiv \frac{2 \mu}{\sqrt{\pi}} \int_{0}^{x} d s \exp \left(-\mu^{2} s^{2}\right)
$$

with $\Phi(z)$ as the error function. The error function of imaginary argument has a close relation to the Dawson integral denoted by $F(z)$ in $(4.18)$. The function $h_{0}(q / \sqrt{\hbar}) / \hbar^{1 / 4}$ is the normalized solution of the wave equation for the harmonic oscillator in the ground state that means the eigenstate of the number operator to the eigenvalue $n=0$ in "position" representation. It possesses even parity. The function $g_{0}(q / \sqrt{\hbar})$ is under all nonnormalizable eigenfunctions of the number operator to the eigenvalue $n=0$ the eigenfunction with odd parity.

The normally ordered operator part in Eq.(4.16) is convenient for the calculation of matrix elements in the Fock-state representation and in the coherent-state representation. We begin with the simpler last case. Due to the relation

$$
\left\langle\alpha\left|R(\varphi) \exp \left(-\frac{a^{\dagger}}{\sqrt{2}} \frac{\partial}{\partial x}\right) \exp \left(-\frac{a}{\sqrt{2}} \frac{\partial}{\partial x}\right)(R(\varphi))^{\dagger}\right| \alpha\right\rangle=\exp \left(-\frac{\alpha \mathrm{e}^{-i \varphi}+\alpha^{*} \mathrm{e}^{i \varphi}}{\sqrt{2}} \frac{\partial}{\partial x}\right)
$$

it acts onto the integral in (4.16) as a displacement operator of the argument and one obtains from (4.16) immediately the following reconstruction of the coherent-state quasiprobability from the Radon transform of the Wigner quasiprobability

$$
\begin{aligned}
Q\left(\alpha, \alpha^{*}\right) & \equiv \frac{\langle\alpha|\varrho| \alpha\rangle}{\pi} \\
& =\frac{1}{\pi} \int_{0}^{\pi} d \varphi \int_{-\infty}^{+\infty} d q \breve{W}(\cos \varphi, \sin \varphi ; q) \frac{2 \sqrt{\hbar}}{\pi} \frac{\partial}{\partial q} F\left(\frac{q}{\sqrt{\hbar}}-\frac{\alpha \mathrm{e}^{-i \varphi}+\alpha^{*} \mathrm{e}^{i \varphi}}{\sqrt{2}}\right)
\end{aligned}
$$

Herein, $F(z)$ denotes again the Dawson integral which definition can be taken from Eq.(4.18).

We now consider the reconstruction of the Fock-state matrix elements. First of all, we find for the Fock-state matrix elements of the operator part in (4.16)

$$
S_{m, n}\left(\frac{\partial}{\partial x}\right) \equiv\left\langle m\left|R(\varphi) \exp \left(-\frac{a^{\dagger}}{\sqrt{2}} \frac{\partial}{\partial x}\right) \exp \left(-\frac{a}{\sqrt{2}} \frac{\partial}{\partial x}\right)(R(\varphi))^{\dagger}\right| n\right\rangle
$$




$$
\begin{aligned}
& =\frac{\exp \{i(m-n) \varphi\}}{\sqrt{m ! n !}} \sum_{j=0}^{\{m, n\}} \frac{m ! n !}{j !(m-j) !(n-j) !}\left(-\frac{1}{\sqrt{2}} \frac{\partial}{\partial x}\right)^{m+n-2 j} \\
& =\exp \{i(m-n) \varphi\} \sqrt{\frac{n !}{m !}}\left(-\frac{1}{\sqrt{2}} \frac{\partial}{\partial x}\right)^{m-n} L_{n}^{m-n}\left(-\frac{1}{2} \frac{\partial^{2}}{\partial x^{2}}\right)
\end{aligned}
$$

where $L_{n}^{m-n}(z)$ denote the associated Laguerre polynomials. By forming the Fock-state matrix elements in (4.16) and by inserting then (4.23) one obtains the following structure of the reconstruction formula

$$
\langle m|\varrho| n\rangle=\frac{1}{\pi} \int_{0}^{\pi} d \varphi \int_{-\infty}^{+\infty} d q \breve{W}(\cos \varphi, \sin \varphi ; q) \exp \{i(m-n) \varphi\} F_{m, n}\left(\frac{q}{\sqrt{\hbar}}\right)
$$

with functions $F_{m, n}(x)$ defined in the following way

$$
\begin{aligned}
F_{m, n}(x) & \equiv \frac{\partial}{\partial x}\left\{\frac{1}{\sqrt{m ! n !}} \sum_{j=0}^{\{m, n\}} \frac{m ! n !}{j !(m-j) !(n-j) !}\left(-\frac{1}{\sqrt{2}} \frac{\partial}{\partial x}\right)^{m+n-2 j} h_{0}(x) g_{0}(x)\right\} \\
& =\frac{1}{\sqrt{m ! n !}} \sum_{j=0}^{\{m, n\}} \frac{m ! n !}{j !(m-j) !(n-j) !}\left(-\frac{1}{\sqrt{2}} \frac{\partial}{\partial x}\right)^{m+n-2 j} F_{0,0}(x)=F_{n, m}(x)
\end{aligned}
$$

The functions $\exp \{i(m-n) \varphi\} F_{m, n}(x)$ are called "pattern functions" for the reconstruction of the Fock-state matrix elements [12-14]. However, contrary to the cited papers we have defined them with a factor $\pi$ larger because then the integral over the angle $\varphi$ in (4.24) with $1 / \pi$ in front can be considered as an avering over the angle. The angle-dependent part is explicitly splitted in (4.24) from the more complicated position-dependent part. The pattern functions $F_{m, n}(x)$ are symmetrical in the indices by definition (4.25).

Let us consider the explicit representation of the pattern functions by functions of the parabolic cylinder $D_{\nu}(z)$. Due to the relations

$$
\left( \pm \frac{i}{\sqrt{2}}\left(x-\frac{\partial}{\partial x}\right)\right)^{n} D_{\nu}( \pm i \sqrt{2} x)=\frac{\Gamma(\nu+1)}{\Gamma(\nu-n+1)} D_{\nu-n}( \pm i \sqrt{2} x)
$$

following from basic definitions of these functions one obtains by accomplishing the differentiations in (4.25) and taking into account (4.18) and (4.19)

$$
\begin{aligned}
F_{m, n}(x)= & \exp \left(-\frac{x^{2}}{2}\right) 2 \sqrt{m ! n !} \sum_{j=0}^{\{m, n\}} \frac{(-1)^{j}(m+n+1-2 j) !}{j !(m-j) !(n-j) !} \\
& \times \frac{i^{m+n}}{2}\left\{D_{-(m+n+2-2 j)}(i \sqrt{2} x)+(-1)^{m+n} D_{-(m+n+2-2 j)}(-i \sqrt{2} x)\right\} .
\end{aligned}
$$

This is near to the representation derived in [14] but the calculations are made there more generally for the reconstruction of the matrix elements in the Fock-state basis from the Radon transform of the $s$-ordered quasiprobabilities. In section 6 we will obtain an alternative representation of the pattern functions in form of a series over even or odd Hermite polynomials. 
We now derive a specific structure of the pattern functions as derivatives of the products of a normalizable with a nonnormalizable wave function of the harmonic oscillator [18-21]. The operator in (4.23) acts onto the product $h_{0}(x) g_{0}(x)$ as an excitation operator. The creation operator $a^{\dagger}$ can be substituted in application to wave functions $\langle q \mid \psi\rangle \equiv \psi(q)$ by

$$
a^{\dagger}=\frac{Q-i P}{\sqrt{2 \hbar}} \longrightarrow \frac{1}{\sqrt{2}}\left(\frac{q}{\sqrt{\hbar}}-\sqrt{\hbar} \frac{\partial}{\partial q}\right)=\frac{1}{\sqrt{2}}\left(x-\frac{\partial}{\partial x}\right), \quad x \equiv \frac{q}{\sqrt{\hbar}} .
$$

Therefore, the higher excitations of the eigenfunctions of the number operator can be defined by

$$
\begin{aligned}
& \left(|n\rangle,|n\rangle^{\prime}\right) \equiv \frac{a^{\dagger n}}{\sqrt{n !}}\left(|0\rangle,|0\rangle^{\prime}\right) \longrightarrow\left(h_{n}(x), g_{n}(x)\right) \equiv \frac{1}{\sqrt{2^{n} n !}}\left(x-\frac{\partial}{\partial x}\right)^{n}\left(h_{0}(x), g_{0}(x)\right) \\
& \int_{-\infty}^{+\infty} d x h_{m}(x) h_{n}(x)=\delta_{m, n}, \quad W\left(h_{n}(x), g_{n}(x)\right)=2
\end{aligned}
$$

where $|n\rangle^{\prime}$ is the abstract notation for the nonnormalizable eigenstates of the number operator with parity $(-1)^{n+1}$. The functions $h_{n}(x)$ are the orthonormalized Hermite functions or with the substitution $x=q / \sqrt{\hbar}$ and the absent normalization factor $1 / \hbar^{(1 / 4)}$ the wave functions $\langle q \mid n\rangle$ given in (4.5). In the same way, the annihilation operator $a$ in "position" representation can be represented according to

$$
a=\frac{Q+i P}{\sqrt{2 \hbar}} \longrightarrow \frac{1}{\sqrt{2}}\left(\frac{q}{\sqrt{\hbar}}+\sqrt{\hbar} \frac{\partial}{\partial q}\right)=\frac{1}{\sqrt{2}}\left(x+\frac{\partial}{\partial x}\right) .
$$

However, there is a very important difference concerning the action of the operator $a$ to the vacuum state $|0\rangle$ and to the state $|0\rangle$ ', in "position" representation

$$
\begin{array}{ll}
\frac{1}{\sqrt{2}}\left(x+\frac{\partial}{\partial x}\right) h_{0}(x)=0, & \frac{1}{\sqrt{2}}\left(x+\frac{\partial}{\partial x}\right) g_{0}(x)=\pi^{\frac{1}{4}} \sqrt{2} \exp \left(\frac{x^{2}}{2}\right) \equiv g_{-1}(x), \\
\frac{1}{\sqrt{2}}\left(x-\frac{\partial}{\partial x}\right) g_{-1}(x)=0, & \frac{1}{\sqrt{2}}\left(x-\frac{\partial}{\partial x}\right) \frac{1}{\sqrt{2}}\left(x+\frac{\partial}{\partial x}\right) g_{-1}(x)=-g_{-1}(x) .
\end{array}
$$

Obviously, by repeated action of the annihilation operator in position representation onto the state $g_{-1}(x)$ one can obtain nonnormalizable eigenstates $g_{-n}(x)$ of the number operator to the eigenvalues $-n$ whereas for the normalizable eigenstates the corresponding series is truncated below the vacuum state $h_{0}(x)$. We do not investigate this here more in detail but show that the asymmetry in the action of the annihilation operator onto $h_{0}(x)$ and $g_{0}(x)$ affects an assymmetry in the representation of the pattern functions by the series $h_{m}(x)$ and $g_{n}(x)$.

We now prove that

$$
F_{m, n}(x)=\frac{\partial}{\partial x}\left\{h_{m}(x) g_{n}(x)\right\}, \quad(m \leq n+1), \quad\left(F_{m, n}(x)=F_{n, m}(x)\right) .
$$


The proof of this formula can be made by complete induction using the recursion relations

$$
\begin{aligned}
& S_{m, n+1}(u)=\frac{1}{\sqrt{n+1}}\left\{-\mathrm{e}^{-i \varphi} \frac{u}{\sqrt{2}} S_{m, n}(u)+\sqrt{n} S_{m-1, n}(u)\right\}, \\
& S_{m+1, n}(u)=\frac{1}{\sqrt{m+1}}\left\{-\mathrm{e}^{i \varphi} \frac{u}{\sqrt{2}} S_{m, n}(u)+\sqrt{n} S_{m, n-1}(u)\right\},
\end{aligned}
$$

following from (4.23). The relation (4.32) is obviously true for $m=0$ and arbitrary $n$ because of

$$
\frac{\partial}{\partial x} \frac{1}{\sqrt{2^{n} n !}}\left(-\frac{\partial}{\partial x}\right)^{n}\left\{h_{0}(x) g_{0}(x)\right\}=\frac{\partial}{\partial x} h_{0}(x) \frac{1}{\sqrt{2^{n} n !}}\left(x-\frac{\partial}{\partial x}\right)^{n} g_{0}(x)=\frac{\partial}{\partial x}\left\{h_{0}(x) g_{n}(x)\right\} \text {. }
$$

On the other side, however, one finds

$$
\begin{aligned}
& \frac{\partial}{\partial x} \frac{1}{\sqrt{2^{1} 1 !}}\left(-\frac{\partial}{\partial x}\right)\left\{h_{0}(x) g_{0}(x)\right\}=\frac{\partial}{\partial x}\left\{h_{1}(x) g_{0}(x)-\sqrt{2}\right\} \\
& \frac{\partial}{\partial x} \frac{1}{\sqrt{2^{2} 2 !}}\left(-\frac{\partial}{\partial x}\right)^{2}\left\{h_{0}(x) g_{0}(x)\right\}=\frac{\partial}{\partial x}\left\{h_{2}(x) g_{0}(x)-\sqrt{2} x\right\} .
\end{aligned}
$$

This means that (4.32) is not generally true for all $(m, n=0)$ without restriction. Let us give the proof of (4.32) by complete induction. Suppose that it is true for a certain $(m, n)$. Then by using the recursion relation (4.33) one can prove without difficulties that it is also true for $(m, n+1)$. We do not explicitly write this down because we will write down the induction from $(m, n)$ to $(m+1, n)$ which is very similar but shows in addition a specific difficulty. By using (4.33) one finds by induction from $(m, n)$ to $(m+1, n)$

$$
\begin{aligned}
& \frac{\partial}{\partial x} S_{m+1, n}\left(\frac{\partial}{\partial x}\right)\left\{h_{0}(x) g_{0}(x)\right\} \\
= & \frac{\partial}{\partial x} \frac{\mathrm{e}^{i(m+1-n) \varphi}}{\sqrt{m+1}}\left\{g_{n}(x) \frac{1}{\sqrt{2}}\left(x-\frac{\partial}{\partial x}\right) h_{m}(x)-h_{m}(x)\left(\frac{1}{\sqrt{2}}\left(x+\frac{\partial}{\partial x}\right) g_{n}(x)-\sqrt{n} g_{n-1}(x)\right)\right\} \\
= & \mathrm{e}^{i(m+1-n) \varphi} \frac{\partial}{\partial x}\left\{h_{m+1}(x) g_{n}(x)-\delta_{n, 0} h_{m}(x) g_{-1}(x)\right\}
\end{aligned}
$$

where the relations (4.29) and (4.30) are applied. The sum term proportional to $\delta_{n, 0}$ in brackets which have its origin in the relations (4.31) prevents the full proof of (4.32) for all $(m+1, n)$. However, if one raises at once $n$ to $n+1$ that means if one makes the complete induction from $(m, n)$ to $(m+1, n+1)$ then the proof makes no difficulties because of $\delta_{n+1,0}=0$ for all nonnegative $n$. This means that the first relation in Eq.(4.32) is proved with the restriction $m \leq n+1$ because it is right for $(m=0, n)$ and $(m=1, n)$. If we do not consider the restriction $m \leq n+1$ then one obtains new pattern functions by the first of the relations in Eq.(4.32) which do not coincide with $F_{m, n}(x)$ defined by (4.25) or (4.27) for $m>n+1$ but can be used by full rights as equivalent pattern functions. We investigate this nonuniqueness of the pattern functions for the reconstruction of the Fock-state matrix elements furthermore in sections 6 and 7 . The pattern functions given by (4.25) or (4.27) possess the advantage that they vanish in infinity. 


\section{Reconstruction of the normally ordered moments from the Radon transform of the Wigner quasiprobability}

The normally ordered moments $\left\langle a^{\dagger k} a^{l} \varrho\right\rangle$ can be directly reconstructed from the Radon transform of the Wigner quasiprobability without the intermediate calculation of the matrix elements in the Fock-state representation as found by Richter [24. A very simple derivation of this reconstruction is given in [25] where is also shown that the integration over the angle in the Radon transform made in [24] is not necessary and can be substituted by certain summations over discrete angles that leads to more basic formulas.

The starting point for the reconstruction of the normally ordered moments is the following representation of the density operator by the normally ordered moments derived in [63-65]

$$
\varrho=\sum_{k=0}^{\infty} \sum_{l=0}^{\infty} a_{k, l}\left\langle a^{\dagger k} a^{l} \varrho\right\rangle, \quad a_{k, l} \equiv \sum_{j=0}^{\{k, l\}} \frac{(-1)^{j}|l-j\rangle\langle k-j|}{j ! \sqrt{(k-j) !(l-j) !}} .
$$

It can be easily obtained from the normally ordered expansion of the basic Fock-state operators $|m\rangle\langle n|$ in powers of the boson operators [71]. For the Radon transform of the Wigner quasiprobability in the form $\breve{W}(\cos \varphi, \sin \varphi ; q)$ one finds from $(5.1)$

$$
\breve{W}(\cos \varphi, \sin \varphi ; q)=\langle q ; \varphi|\varrho| q ; \varphi\rangle=\sum_{k=0}^{\infty} \sum_{l=0}^{\infty}\left\langle q ; \varphi\left|a_{k, l}\right| q ; \varphi\right\rangle\left\langle a^{\dagger k} a^{l} \varrho\right\rangle
$$

and with the explicit form of the position representation of the number states by Hermite functions

$$
\begin{aligned}
\left\langle q ; \varphi\left|a_{k, l}\right| q ; \varphi\right\rangle & =\frac{1}{\sqrt{\pi \hbar}} \exp \left(-\frac{q^{2}}{\hbar}\right) \frac{e^{i(k-l) \varphi}}{\sqrt{2^{k+l}} k ! l !} \sum_{j=0}^{\{k, l\}} \frac{(-2)^{j} k ! l !}{j !(k-j) !(l-j) !} H_{k-j}\left(\frac{q}{\sqrt{\hbar}}\right) H_{l-j}\left(\frac{q}{\sqrt{\hbar}}\right) \\
& =\frac{1}{\sqrt{\pi \hbar}} \exp \left(-\frac{q^{2}}{\hbar}\right) \frac{e^{i(k-l) \varphi}}{\sqrt{2^{k+l}} k ! l !} H_{k+l}\left(\frac{q}{\sqrt{\hbar}}\right) .
\end{aligned}
$$

Here we used a known identity for finite sums over products of Hermite polynomials ( see Eq.10.13. (36) in [63] ) which can be proved by complete induction. By multiplication of 
Eq. (5.3) by $H_{n}(q / \sqrt{\hbar})$ and by using the well-known completeness relations for the Hermite functions one finds

$$
\begin{aligned}
& \frac{1}{\sqrt{2^{n}}} \int_{-\infty}^{+\infty} d q \breve{W}(\cos \varphi, \sin \varphi ; q) H_{n}\left(\frac{q}{\sqrt{\hbar}}\right)=\sum_{k=0}^{n} \frac{n !}{k !(n-k) !} e^{i(2 k-n) \varphi}\left\langle a^{\dagger k} a^{n-k} \varrho\right\rangle \\
= & \left\langle\mathcal{N}\left\{\left(a e^{-i \varphi}+a^{\dagger} e^{i \varphi}\right)^{n}\right\} \varrho\right\rangle=\left(\sqrt{\frac{2}{\hbar}}\right)^{n}\left\langle\mathcal{N}\left\{(Q(\varphi))^{n}\right\} \varrho\right\rangle .
\end{aligned}
$$

The symbol $\mathcal{N}\{\ldots\}$ denotes normal ordering of the content in braces. The system of equations (5.4) for the normally ordered moments $\left\langle a^{\dagger k} a^{n-k} \varrho\right\rangle$ can be solved by using the properties of the solutions of the circle division problem. The solutions $z$ for the division of the unit circle in $n+1$ equal sectors ( harmonic division )

$$
0=z^{n+1}-1=(z-1) \sum_{m=0}^{n} z^{m}, \quad z=\exp \left(i s \frac{2 \pi}{n+1}\right), \quad s=0,1, \ldots, n,
$$

possess the property

$$
\sum_{m=0}^{n} z^{m}=\sum_{m=0}^{n} \exp \left(i m s \frac{2 \pi}{n+1}\right)=(n+1) \delta_{s, 0}
$$

By using these orthogonality relations of the solutions of the circle division problem one finds from Eq.(5.4)

$$
\begin{aligned}
\left\langle a^{\dagger k} a^{l} \varrho\right\rangle= & \frac{k ! l !}{(k+l+1) !} \sum_{m=0}^{k+l} \exp \left\{-i(k-l)\left(\varphi_{0}+\frac{m \pi}{k+l+1}\right)\right\} \frac{1}{\sqrt{2^{k+l}}} \\
& \times \int_{-\infty}^{+\infty} d q \breve{W}\left\{\cos \left(\varphi_{0}+\frac{m \pi}{k+l+1}\right), \sin \left(\varphi_{0}+\frac{m \pi}{k+l+1}\right) ; q\right\} H_{k+l}\left(\frac{q}{\sqrt{\hbar}}\right) .
\end{aligned}
$$

Herein, $\varphi_{0}$ denotes an arbitrary initial angle. This formula is the only place we know where our notations of the Radon transform seem to be a little bulky. By integration over the arbitrary initial angle $\varphi_{0}$ one obtains 24, 25]

$$
\left\langle a^{\dagger k} a^{l} \varrho\right\rangle=\frac{k ! l !}{(k+l) !} \frac{1}{\pi} \int_{0}^{\pi} d \varphi \exp \{-i(k-l) \varphi\} \frac{1}{\sqrt{2^{k+l}}} \int_{-\infty}^{+\infty} d q \breve{W}(\cos \varphi, \sin \varphi ; q) H_{k+l}\left(\frac{q}{\sqrt{\hbar}}\right) .
$$

It is interesting to mention that from a technical point of view it is mostly more simple to make first the integration over the variable $q$ in this formula for simple cases of Radon transforms such as, for example, for squeezed coherent states whereas the direct integration over the angle of the Radon transform multiplied by phase factors is connected with considerable technical difficulties.

It arises the question whether solutions of Eq.(5.4) with unequal divisions of the unit circle are possible or not. Such solutions are, in principle, possible if one uses $n+1$ inequivalent 
discrete angles ( pairs of angles $\varphi$ and $\varphi+\pi$ are equivalent ). However, it seems to be difficult to find general solutions for arbitrary $n$ in explicit form. For low orders of $n$ it is possible to solve the system of algebraic equations obtained for discrete angles. In such a way one finds for the first-order moments

$$
\begin{aligned}
\langle a \varrho\rangle= & \frac{1}{2 \sqrt{2}} \int_{-\infty}^{+\infty} d q\left\{\frac{\exp \left(i \varphi_{1}\right) \breve{W}\left(\cos \varphi_{0}, \sin \varphi_{0} ; q\right)}{i \sin \left(\varphi_{1}-\varphi_{0}\right)}+\frac{\exp \left(i \varphi_{0}\right) \breve{W}\left(\cos \varphi_{1}, \sin \varphi_{1} ; q\right)}{i \sin \left(\varphi_{0}-\varphi_{1}\right)}\right\} \\
& \times H_{1}\left(\frac{q}{\sqrt{\hbar}}\right), \quad\left\langle a^{\dagger} \varrho\right\rangle=\langle a \varrho\rangle^{*}, \quad H_{1}(x)=2 x,
\end{aligned}
$$

and for the second-order moments

$$
\begin{aligned}
\left\langle a^{2} \varrho\right\rangle= & \frac{1}{8} \int_{-\infty}^{+\infty} d q\left\{\frac{\exp \left(i\left(\varphi_{1}+\varphi_{2}\right)\right) \breve{W}\left(\cos \varphi_{0}, \sin \varphi_{0} ; q\right)}{\sin \left(\varphi_{1}-\varphi_{0}\right) \sin \left(\varphi_{0}-\varphi_{2}\right)}\right. \\
& +\frac{\exp \left(i\left(\varphi_{2}+\varphi_{0}\right)\right) \breve{W}\left(\cos \varphi_{1}, \sin \varphi_{1} ; q\right)}{\sin \left(\varphi_{1}-\varphi_{0}\right) \sin \left(\varphi_{2}-\varphi_{1}\right)} \\
& \left.+\frac{\exp \left(i\left(\varphi_{0}+\varphi_{1}\right)\right) \breve{W}\left(\cos \varphi_{2}, \sin \varphi_{2} ; q\right)}{\sin \left(\varphi_{2}-\varphi_{1}\right) \sin \left(\varphi_{0}-\varphi_{2}\right)}\right\} H_{2}\left(\frac{q}{\sqrt{\hbar}}\right), \\
\left\langle a^{\dagger} a \varrho\right\rangle= & -\frac{1}{8} \int_{-\infty}^{+\infty} d q\left\{\frac{\cos \left(\varphi_{2}-\varphi_{1}\right) \breve{W}\left(\cos \varphi_{0}, \sin \varphi_{0} ; q\right)}{\sin \left(\varphi_{1}-\varphi_{0}\right) \sin \left(\varphi_{0}-\varphi_{2}\right)}\right. \\
& +\frac{\cos \left(\varphi_{0}-\varphi_{2}\right) \breve{W}\left(\cos \varphi_{1}, \sin \varphi_{1} ; q\right)}{\sin \left(\varphi_{1}-\varphi_{0}\right) \sin \left(\varphi_{2}-\varphi_{1}\right)} \\
& \left.+\frac{\cos \left(\varphi_{1}-\varphi_{0}\right) \breve{W}\left(\cos \varphi_{2}, \sin \varphi_{2} ; q\right)}{\sin \left(\varphi_{2}-\varphi_{1}\right) \sin \left(\varphi_{0}-\varphi_{2}\right)}\right\} H_{2}\left(\frac{q}{\sqrt{\hbar}}\right), \\
\left\langle a^{\dagger} \varrho\right\rangle= & \left\langle a^{2} \varrho\right\rangle^{*}, \quad H_{2}(x)=4 x^{2}-2 .
\end{aligned}
$$

It is easy to specify these solutions. For example, for $\varphi_{0}=0, \varphi_{1}=\pi / 4, \varphi_{2}=\pi / 2$ it follows from (5.10)

$$
\begin{aligned}
\left\langle a^{2} \varrho\right\rangle & =\frac{1}{8} \int_{-\infty}^{+\infty} d q\left\{(1-i) \breve{W}(1,0 ; q)+i 2 \breve{W}\left(\frac{\sqrt{2}}{2}, \frac{\sqrt{2}}{2} ; q\right)-(1+i) \breve{W}(0,1 ; q)\right\} H_{2}\left(\frac{q}{\sqrt{\hbar}}\right), \\
\left\langle a^{\dagger} a \varrho\right\rangle & =\frac{1}{8} \int_{-\infty}^{+\infty} d q\{\breve{W}(1,0 ; q)+\breve{W}(0,1 ; q)\} H_{2}\left(\frac{q}{\sqrt{\hbar}}\right),
\end{aligned}
$$

and for harmonic division $\varphi_{0}=0, \varphi_{1}=\pi / 3, \varphi_{2}=2 \pi / 3$ 


$$
\begin{aligned}
\left\langle a^{2} \varrho\right\rangle= & \frac{1}{6} \int_{-\infty}^{+\infty} d q\left\{\breve{W}(1,0 ; q)-\frac{1-i \sqrt{3}}{2} \breve{W}\left(\frac{1}{2}, \frac{\sqrt{3}}{2} ; q\right)-\frac{1+i \sqrt{3}}{2} \breve{W}\left(-\frac{1}{2}, \frac{\sqrt{3}}{2} ; q\right)\right\} \\
& \times H_{2}\left(\frac{q}{\sqrt{\hbar}}\right), \\
\left\langle a^{\dagger} a \varrho\right\rangle= & \frac{1}{12} \int_{-\infty}^{+\infty} d q\left\{\breve{W}(1,0 ; q)+\breve{W}\left(\frac{1}{2}, \frac{\sqrt{3}}{2} ; q\right)+\breve{W}\left(-\frac{1}{2}, \frac{\sqrt{3}}{2} ; q\right)\right\} H_{2}\left(\frac{q}{\sqrt{\hbar}}\right) .
\end{aligned}
$$

We are not sure to what extent Eqs.(5.9) and (5.10) can be explicitly generalized. The harmonic division has the disadvantage that one must change the division when going from moments of the $n$-th order to moments of $(n+1)$-th order. If one is only interested up to moments of the 4 -th order then one can take the harmonic division in 5 angles according to $\varphi_{0}+(m \pi) / 5$ with $m=0,1, \ldots, 4$ and can use for the lower moments subsets of these angles corresponding to anharmonic divisions. In photon statistics one considers only moments $\left\langle N^{l} \varrho\right\rangle$ corresponding to linear combinations of normally ordered moments $\left\langle a^{\dagger k} a^{k} \varrho\right\rangle$ up to the order $l$. It is quite possible that some other special solutions of the systems of equations (5.4) corresponding to anharmonic divisions can be found. 


\section{Alternative method of the reconstruction of the matrix elements}

The matrix elements of the density operator in Fock-state representation can be also reconstructed from the normally ordered moments of the density operator. If we use the reconstruction of the normally ordered moments from the Radon transform that was discussed in the last section as an intermediate step to the reconstruction of the matrix elements then we arrive at a new relatively simple alternative representation of the pattern functions. We now consider the derivation.

From the representation of the density operator by the normally ordered moments in Eq.(4.1) one obtains by forming the matrix elements with Fock states

$$
\begin{aligned}
\langle m|\varrho| n\rangle & =\sum_{k=0}^{\infty} \sum_{l=0}^{\infty}\left\langle m\left|a_{k, l}\right| n\right\rangle\left\langle a^{\dagger k} a^{l} \varrho\right\rangle \\
& =\sum_{k=0}^{\infty} \sum_{l=0}^{\infty} \sum_{j=0}^{\{k, l\}} \frac{(-1)^{j}\langle m \mid l-j\rangle\langle k-j \mid n\rangle}{j ! \sqrt{(k-j) !(l-j) !}}\left\langle a^{\dagger k} a^{l} \varrho\right\rangle \\
& =\frac{1}{\sqrt{m ! n !}} \sum_{j=0}^{\infty} \frac{(-1)^{j}}{j !}\left\langle a^{\dagger n+j} a^{m+j} \varrho\right\rangle .
\end{aligned}
$$

By inserting the reconstruction formula for the normally ordered moments from the Radon transform in the form averaged over the angle (5.8) one finds after changing the order of summation and integration

$$
\begin{aligned}
\langle m|\varrho| n\rangle= & \frac{1}{\pi} \int_{0}^{\pi} d \varphi \exp \{i(m-n) \varphi\} \int_{-\infty}^{+\infty} d q \breve{W}(\cos \varphi, \sin \varphi ; q) \\
& \times \frac{1}{\sqrt{2^{m+n} m ! n !}} \sum_{j=0}^{\infty} \frac{(m+j) !(n+j) !}{j !(m+n+2 j) !}\left(-\frac{1}{2}\right)^{j} H_{m+n+2 j}\left(\frac{q}{\sqrt{\hbar}}\right) \\
\equiv & \frac{1}{\pi} \int_{0}^{\pi} d \varphi \exp \{i(m-n) \varphi\} \int_{-\infty}^{+\infty} d q \breve{W}(\cos \varphi, \sin \varphi ; q) F_{m, n}^{\prime}\left(\frac{q}{\sqrt{\hbar}}\right) .
\end{aligned}
$$

This means that we derived a representation of the pattern functions $F_{m, n}^{\prime}(x)$ by an infinite series over Hermite polynomials of even or odd order in increasing steps of 2 in the indices with some coefficients and in dependence on the even or odd order of $m+n$

$$
F_{m, n}^{\prime}(x)=\frac{1}{\sqrt{2^{m+n} m ! n !}} \sum_{j=0}^{\infty} \frac{(m+j) !(n+j) !}{j !(m+n+2 j) !}\left(-\frac{1}{2}\right)^{j} H_{m+n+2 j}(x)
$$




$$
=\frac{\partial}{\partial x}\left\{\frac{1}{2 \sqrt{2^{m+n} m ! n !}} \sum_{j=0}^{\infty} \frac{(m+j) !(n+j) !}{j !(m+n+2 j+1) !}\left(-\frac{1}{2}\right)^{j} H_{m+n+2 j+1}(x)\right\} .
$$

The second representation in (6.3) by a derivative of a function with respect to $q$ was made because it corresponds to the general structure of the pattern functions as derived in [1820] where the content in braces can be represented as a product of the only normalizable eigenfunction of the number operator with a nonnormalizable eigenfunction of this operator or linear combinations of such products ( superpositions of the normalizable with each nonnormalizable eigenfunction give in every case nonnormalizable eigenfunctions, however, with no determined parity, in general ). We wrote $F_{m, n}^{\prime}(x)$ for the pattern functions because they must not necessarily be identical with the pattern functions $F_{m, n}(x)$ derived in Eq.(4.27). We now investigate this new phenomenon of the nonuniqueness of the pattern functions.

In the last section it was found in Eq.(5.4) that the multiplication of the Radon transform with a Hermite polynomial $H_{s}(q / \sqrt{\hbar})$ and its integration over $q$ leads to a linear combination of $(s+1)$ normally ordered moments of degree $s$ multiplied by some binomials coefficients and phase factors $\mathrm{e}^{i(2 k-s) \varphi}$ with $k=0,1, \ldots, s$. In the reconstruction formula of the Fockstate matrix elements from the Radon transform of the Wigner quasiprobability (4.24) the pattern functions $F_{m, n}(x)$ have to be multiplied by phase factors $\mathrm{e}^{i(m-n) \varphi}$ and integrated over $\varphi$ from 0 to $\pi$. Because of

$$
\frac{1}{\pi} \int_{0}^{\pi} d \varphi \exp (i 2 l \varphi)=\delta_{l, 0}
$$

two pattern functions $F_{m, n}(x)$ and $F_{m, n}^{\prime}(x)$ connected by

$$
F_{m, n}^{\prime}(x)=F_{m, n}(x)+\sum_{k=1}^{\left[\left|\frac{m-n}{2}\right|\right]} c_{k} H_{|m-n|-2 k}(x),
$$

where $[\nu]$ denotes the integer part of $\nu$ and where $c_{k}$ are arbitrary coefficients, lead to the same result for the reconstruction of the Fock-state matrix elements $\langle m|\varrho| n\rangle$ and are equivalent. It is not proved that (6.5) is the most general form of the nonuniqueness of the pattern functions but it seems so and it is the only form of the nonuniqueness which plays a role in the present paper. The pattern functions obtained in (4.27) become identical with the pattern functions in (6.3) if we only formally extend the summation to all possible negative summation indices $j$, i.e.

$$
\begin{aligned}
F_{m, n}(x)= & \frac{1}{\sqrt{2^{m+n} m ! n !}} \sum_{j=-\left[\frac{m+n}{2}\right]}^{\infty} \frac{(m+j) !(n+j) !}{j !(m+n+2 j) !}\left(-\frac{1}{2}\right)^{j} H_{m+n+2 j}(x) \\
= & F_{m, n}^{\prime}(x) \\
& +\frac{1}{\sqrt{2^{|m-n|} m ! n !}} \sum_{k=1}^{\left[\left|\frac{m-n}{2}\right|\right]} \frac{(k-1+\{m, n\}) !(|m-n|-k) !}{(k-1) !(|m-n|-2 k) !}(-2)^{k} H_{|m-n|-2 k}(x),
\end{aligned}
$$


where $\{m, n\} \equiv \operatorname{Min}(m, n)$. All investigated special cases are in agreement with this statement but the general proof is not made up to now. In special cases one finds

$$
\begin{aligned}
& F_{n, n}(x)=F_{n, n}^{\prime}(x), \quad F_{n+1, n}(x)=F_{n+1, n}^{\prime}(x), \\
& F_{n+2, n}(x)=F_{n+2, n}^{\prime}(x)-\sqrt{\frac{n !}{(n+2) !}} H_{0}(x), \quad F_{n+3, n}(x)=F_{n+3, n}^{\prime}(x)-\sqrt{\frac{2 n !}{(n+3) !}} H_{1}(x) .
\end{aligned}
$$

Let us discuss some properties of the pattern functions $F_{m, n}^{\prime}(x)$. First of all, they are symmetric in the indices and possess the parity $(-1)^{m+n}$ as $F_{m, n}(x)$ too

$$
F_{m, n}^{\prime}(x)=F_{n, m}^{\prime}(x), \quad F_{m, n}^{\prime}(-x)=(-1)^{m+n} F_{m, n}^{\prime}(x) .
$$

The summations at the point $x=0$ can be exactly accomplished for not very large even differences $|m-n|$ whereas for all odd differences the corresponding Hermite polynomials in (6.3) vanish for $x=0$. One obtains [72]

$$
F_{n, n}^{\prime}(0)=(-1)^{n} 2, \quad F_{n+2, n}^{\prime}(0)=(-1)^{n+1} \frac{2 n+3}{\sqrt{(n+2)(n+1)}}, \quad F_{n+2 l+1, n}^{\prime}(0)=0
$$

The first maxima and minima of $F_{n, n}^{\prime}(x)=F_{n, n}(x)$ for $x \neq 0$ come for increasing $n$ very near to the values \pm 2 but are not exactly equal to these values [72]. Some similarities in the form of the pattern functions to the corresponding products of wave functions $\langle q \mid m\rangle\langle n \mid q\rangle$ can be explained by the following expansion of products of Hermite polynomials which can be proved by complete induction ( see Eq.10.13 (37) ) in 63 )

$$
\begin{aligned}
& \langle q \mid m\rangle\langle n \mid q\rangle=\frac{1}{\sqrt{\pi \hbar}} \exp \left(-\frac{q^{2}}{\hbar}\right) \frac{1}{\sqrt{2^{m+n} m ! n !}} H_{m}\left(\frac{q}{\sqrt{\hbar}}\right) H_{n}\left(\frac{q}{\sqrt{\hbar}}\right), \\
& H_{m}(x) H_{n}(x)=\sum_{j=0}^{\{m, n\}} \frac{m ! n !}{j !(m-j) !(n-j) !} 2^{j} H_{m+n-2 j}(x) .
\end{aligned}
$$

The first polynomial terms in the expansions in (6.3) and (6.10) are both $H_{m+n}(x)$. Contrary to (6.3), the next terms in the expansion in (6.10) are proportional to Hermite polynomials with indices decreasing in steps of 2 but if the normalized Gaussian function $\exp \left(-x^{2}\right) / \sqrt{\pi}$ is included into the expansion in terms of Hermite polynomials, then we get a more complicated formula which we do not derive here. Thus one has similarities in the representation of the products of wave functions and corresponding pattern functions by expansions in Hermite polynomials.

The representation (6.3) of the pattern functions is appropriate for their calculation by a computer with good accuracy for not too large arguments ( say $|x|<5$ with $\sim 50$ initial sum terms and not too high $(m, n)$, e.g., for $m=n=0$ one obtains by 15 initial terms an approximation where the deviations begin for $|x| \approx 5$ ). For large values of the argument one can use the highest power in the Hermite polynomials $H_{n}(x)$ that means $(2 x)^{n}$ as an 
approximation. It is interesting that the Hermite polynomials $H_{n}(x)$ can be obtained from these asymptotic functions $(2 x)^{n}$ by the following convolution [73, 74]

$$
H_{n}(x)=\exp \left(-\frac{1}{4} \frac{\partial^{2}}{\partial x^{2}}\right)(2 x)^{n}=\frac{1}{\sqrt{-\pi}} \exp \left(x^{2}\right) *(2 x)^{n} .
$$

One can prove by Taylor series expansion of the exponential function that one obtains by this convolution the explicit representation of the Hermite polynomials. By applying this to Eq.(6.3) one finds

$$
F_{m, n}^{\prime}(x)=\exp \left(-\frac{1}{4} \frac{\partial^{2}}{\partial x^{2}}\right) \frac{(\sqrt{2} x)^{m+n}}{\sqrt{m ! n !}} \sum_{j=0}^{\infty} \frac{(m+j) !(n+j) !}{j !(m+n+2 j) !}\left(-2 x^{2}\right)^{j} .
$$

If we omit the convolution operator $\exp \left(-(1 / 4) \partial^{2} / \partial x^{2}\right)$ in this relation we get an asymptotic representation of the pattern functions for large values $|x|$.

If we use the explicit representation of the Hermite polynomials in the pattern function $F_{0,0}^{\prime}(x)=F_{0,0}(x)$ we arrive, after reordering of the sum terms and accomplishing one of the sums, at the following Taylor series representation

$$
\begin{aligned}
F_{0,0}(x) & =\sum_{j=0}^{\infty} \frac{j !}{(2 j) !}\left(-\frac{1}{2}\right)^{j} H_{2 j}(x) \\
& =\frac{\partial}{\partial x} \frac{1}{2} \sum_{j=0}^{\infty} \frac{j !}{(2 j+1) !}\left(-\frac{1}{2}\right)^{j} H_{2 j+1}(x) \\
& =2 \sum_{k=0}^{\infty} \frac{(-1)^{k} k !}{(2 k) !}(2 x)^{2 k} .
\end{aligned}
$$

This can be also represented in the forms

$$
\begin{aligned}
F_{0,0}(x) & =\frac{\partial}{\partial x}\left\{2 \exp \left(-x^{2}\right) \int_{0}^{x} d t \exp \left(t^{2}\right)\right\} \\
& =\frac{\partial}{\partial x}\left\{\sqrt{2} \exp \left(-\frac{x^{2}}{2}\right) \frac{i}{2}\left\{D_{-1}(i \sqrt{2} x)-D_{-1}(-i \sqrt{2} x)\right\}\right\} \\
& =\exp \left(-\frac{x^{2}}{2}\right)\left\{D_{-2}(i \sqrt{2} x)+D_{-2}(-i \sqrt{2} x)\right\} .
\end{aligned}
$$

where $D_{\nu}(z)$ denotes the functions of the parabolic cylinder. Let us show that the content in braces in Eq.(6.3) is proportional to the product of the normalizable eigenfunction of order $m$ and the nonnormalizable eigenfunction of order $n$. For this purpose we make the following transformation

$$
\begin{aligned}
& \left\{\left(x-\frac{\partial}{\partial x}\right)^{m} \exp \left(-\frac{x^{2}}{2}\right)\right\}\left\{\left(x-\frac{\partial}{\partial x}\right)^{n} 2 \exp \left(-\frac{x^{2}}{2}\right) \int_{0}^{x} d u \exp \left(u^{2}\right)\right\} \\
= & 2 H_{m}(x)\left(-\frac{\partial}{\partial x}\right)^{n}\left\{\exp \left(-x^{2}\right) \int_{0}^{x} d u \exp \left(u^{2}\right)\right\}
\end{aligned}
$$




$$
\begin{aligned}
& =\frac{1}{2} H_{m}(x)\left(-\frac{\partial}{\partial x}\right)^{n} \sum_{k=0}^{\infty} \frac{k !}{(2 k+1) !}\left(-\frac{1}{2}\right)^{k} H_{2 k+1}(x) \\
& =\frac{1}{2} \sum_{j=-\left[\frac{m+n+1}{2}\right]}^{\infty} \frac{(m+j) !(n+j) !}{j !(m+n+2 j+1) !}\left(-\frac{1}{2}\right)^{j} H_{m+n+2 j+1}(x) .
\end{aligned}
$$

In the first step we brought the operator $(x-\partial / \partial x)^{n}$ from the right to the left of the function $\exp \left(-x^{2} / 2\right)$. Then we applied (6.13) and substituted the product of Hermite polynomials by sums over Hermite polynomials according to a known formula ( 63, 10.13 (36) ) which can be proved by complete induction. After rearrangement of the double sum one sum is calculated by using

$$
\sum_{l=0}^{m} \frac{(-1)^{l} m !(n+j+l) !}{l !(m-l) !(n+2 j+1+l) !}=\frac{(m+j) !(n+j) !}{j !(m+n+2 j+1) !}
$$

which can be proved again by complete induction $(m \rightarrow m+1$ because it is true for $m=0$ with arbitrary $n$ and $j$ ). Thus there is directly proved the connection between two essentially different representations of the pattern functions. We think that the representation of the pattern functions by infinite sums over Hermite polynomials possesses some advantages in comparison to the representation by sums over functions of the parabolic cylinder of imaginary argument. 


\section{Differential equations for products of Hermite functions and orthogonality relations}

Initiated by Richter [18] to explain the structure of the pattern functions as first derivatives of the product of the normalizable and one nonnormalizable eigenfunction of the number operator in "position" representation it was found [19, 21] that the most appropriate approach to solve this problem is to consider the differential equation for products of Hermite functions and to establish the orthogonality relations with the help of the adjoint differential equation. This will be presented here. The problem solved in [19] was from one point of view more general because there were considered products of eigenfunctions of more general Hamilton operators for one degree of freedom consisting of the usual part from kinetic energy and of a part from arbitrary potential energy but from the other point of view it was more special because there were considered only products of eigenfunctions to the same eigenvalue. This last restriction is omitted in [21]. The most interesting case for the nondiagonal elements corresponding to products of eigenfunctions to different eigenvalues is, however, the case of the harmonic oscillator because for the most other interesting one-dimensional potentials defined over the whole coordinate axis $R$ one has no degeneracy of energy differences in the eigenvalue spectrum. The multiplication of the Radon transform with phase factors and integration over the angle in case of the harmonic oscillator sorts out the Fock-state matrix elements corresponding to equal energy differences of the energy eigenvalue spectrum.

The differential equations for both series of normalizable and nonnormalizable excitation states $h_{n}(x)$ and $g_{n}(x)$ of the harmonic oscillator in position representation are ( as before we use the abbreviation $x \equiv q / \sqrt{\hbar}$ )

$$
\left\{\frac{\partial^{2}}{\partial x^{2}}-x^{2}+2 n+1\right\} f_{n}(x)=0, \quad \longleftrightarrow \quad\left(\frac{x^{2}}{2}-\frac{1}{2} \frac{\partial^{2}}{\partial x^{2}}\right) f_{n}(x)=\left(n+\frac{1}{2}\right) f_{n}(x)
$$

where $f_{n}(x)$ is written for an arbitrary of the functions $h_{n}(x)$ or $g_{n}(x)$ or their linear combinations. The normalizable functions $h_{n}(x)$ are the Hermite functions. They possess the parity $(-1)^{n}$ and are given up to complex normalization factor in a unique way and are connected with the Hermite polynomials $H_{n}(z)$ and the functions of the parabolic cylinder 
$D_{n}(z)$ as follows

$$
\begin{aligned}
h_{n}(x) & =\frac{1}{\pi^{\frac{1}{4}}} \exp \left(-\frac{x^{2}}{2}\right) \frac{H_{n}(x)}{\sqrt{2^{n} n !}} \\
& =\frac{D_{n}(\sqrt{2} x)}{\pi^{\frac{1}{4}} \sqrt{n !}} \\
& =\frac{\sqrt{2 n !}}{\pi^{\frac{3}{4}}} \frac{i^{n}}{2}\left\{D_{-1-n}(i \sqrt{2} x)+(-1)^{n} D_{-1-n}(-i \sqrt{2} x)\right\} .
\end{aligned}
$$

They satisfy the following orthonormality and completeness relations

$$
\int_{-\infty}^{+\infty} d x h_{m}(x) h_{n}(x)=\delta_{m, n}, \quad \sum_{n=0}^{\infty} h_{n}(x) h_{n}(y)=\delta(x-y) .
$$

All other linearly independent solutions of equation (7.1) are nonnormalizable but among them one can choose $g_{n}(x)$ in a unique way as the solutions with parity $(-1)^{n+1}$ defined in (4.29). The explicit form of these solutions expressed by the functions of the parabolic cylinder is given by

$$
\begin{aligned}
g_{n}(x) & =\pi^{\frac{1}{4}} \sqrt{2 n !} \frac{i^{n+1}}{2}\left\{D_{-1-n}(i \sqrt{2} x)-(-1)^{n} D_{-1-n}(-i \sqrt{2} x)\right\} \\
& =\frac{2 \pi^{\frac{1}{4}}}{\sqrt{2^{n} n !}} \exp \left(\frac{x^{2}}{2}\right)\left\{H_{n}(x) F(x)-\sum_{k=0}^{n-1} \frac{n !}{k !(n-k) !} i^{n-1-k} H_{k}(x) H_{n-1-k}(i x)\right\}
\end{aligned}
$$

where $F(x)$ denotes the Dawson integral defined in (4.18) ( see also (4.20) for the connection to the error function of imaginary argument ). From (7.2) and (7.4) one finds that functions proportional to $D_{-1-n}(i \sqrt{2} x)$ and $D_{-1-n}(-i \sqrt{2} x)$ could be also chosen as two linearly independent solutions of the equations (7.1) but they are both nonnormalizable and do not possess definite parity.

Let us now consider the differential equation for the product $f_{m}(x) g_{n}(x)$ of two functions $f_{m}(x)$ and $g_{n}(x)$ both satisfying differential equations of the form $(7.1)\left(g_{n}(x)\right.$ is here not necessarily the special eigenfunction with parity $\left.(-1)^{n+1}\right)$. Then one finds up to secondorder derivatives

$$
\begin{aligned}
\frac{\partial}{\partial x}\left\{f_{m}(x) g_{n}(x)\right\} & =f_{m}^{(1)}(x) g_{n}(x)+f_{m}(x) g_{n}^{(1)}(x) \\
\frac{\partial^{2}}{\partial x^{2}}\left\{f_{m}(x) g_{n}(x)\right\} & =f_{m}^{(2)}(x) g_{n}(x)+2 f_{m}^{(1)}(x) g_{n}^{(1)}(x)+f_{n}(x) g_{n}^{(2)}(x) \\
& =2 f_{m}^{(1)}(x) g_{n}^{(1)}(x)+2\left(x^{2}-(m+n+1)\right) f_{m}(x) g_{n}(x) .
\end{aligned}
$$

In the next step by further differentiation and by applying (7.1) one finds

$$
\begin{aligned}
& \left\{\frac{\partial^{3}}{\partial x^{3}}-2\left(\left(x^{2}-(m+n+1)\right) \frac{\partial}{\partial x}+\frac{\partial}{\partial x}\left(x^{2}-(m+n+1)\right)\right)\right\} f_{m}(x) g_{n}(x) \\
= & -2(m-n)\left\{f_{m}(x) g_{n}^{(1)}(x)-f_{m}^{(1)}(x) g_{n}(x)\right\},
\end{aligned}
$$


written in a symmetrical form with respect to the derivatives. It is a pure differential equation of third order for $f_{m}(x) g_{n}(x)$ only in case of $m=n$. This is understandable because in this case we can form 3 linearly independent combinations of the basic solutions, for example, $\left(h_{n}(x)\right)^{2}, h_{n}(x) g_{n}(x),\left(g_{n}(x)\right)^{2}$ if $h_{n}(x)$ and $g_{n}(x)$ are two linearly independent solutions of (7.1). For $m \neq n$ one needs a further differentiation of this equation to obtain the following fourth-order differential equation

$$
\begin{aligned}
& \left\{\frac{\partial^{4}}{\partial x^{4}}-2 \frac{\partial}{\partial x}\left(\left(x^{2}-(m+n+1)\right) \frac{\partial}{\partial x}+\frac{\partial}{\partial x}\left(x^{2}-(m+n+1)\right)\right)+4(m-n)^{2}\right\} \\
& \times f_{m}(x) g_{n}(x)=0 .
\end{aligned}
$$

We have here 4 linearly independent solutions of this differential equation as which can be chosen, for example, the products $h_{m}(x) h_{n}(x), h_{m}(x) g_{n}(x), g_{m}(x) h_{n}(x), g_{m}(x) g_{n}(x)$. The operator in the differential equation (7.7) is neither selfadjoint nor anti-selfadjoint. The adjoint differential equation to $(7.7)$ for functions $X_{m, n}(x)$ is

$$
\begin{aligned}
& \left\{\frac{\partial^{4}}{\partial x^{4}}-2\left(\left(x^{2}-(m+n+1)\right) \frac{\partial}{\partial x}+\frac{\partial}{\partial x}\left(x^{2}-(m+n+1)\right)\right) \frac{\partial}{\partial x}+4(m-n)^{2}\right\} \\
& \times X_{m, n}(x)=0 .
\end{aligned}
$$

If we differentiate this equation once more then we get immediately

$$
\begin{aligned}
& \left\{\frac{\partial^{4}}{\partial x^{4}}-2 \frac{\partial}{\partial x}\left(\left(x^{2}-(m+n+1)\right) \frac{\partial}{\partial x}+\frac{\partial}{\partial x}\left(x^{2}-(m+n+1)\right)\right)+4(m-n)^{2}\right\} \\
& \times \frac{\partial}{\partial x} X_{m, n}(x)=0 .
\end{aligned}
$$

This means that $(\partial / \partial x) X_{m, n}(x)$ satisfies the differential equation (7.7) for products of functions $f_{m}(x) g_{n}(x)$ and their linear combinations.

We now make the proper derivation of the orthogonality relations. For this purpose we first write down the following two differential equations according to (7.7) and (7.8)

$$
\begin{aligned}
& \left\{\frac{\partial^{4}}{\partial x^{4}}-2 \frac{\partial}{\partial x}\left(\left(x^{2}-(m+n+1)\right) \frac{\partial}{\partial x}+\frac{\partial}{\partial x}\left(x^{2}-(m+n+1)\right)\right)+4(m-n)^{2}\right\} \\
& \times Y_{m, n}(x)=0, \\
& \left\{\frac{\partial^{4}}{\partial x^{4}}-2\left(\left(x^{2}-(k+l+1)\right) \frac{\partial}{\partial x}+\frac{\partial}{\partial x}\left(x^{2}-(k+l+1)\right)\right) \frac{\partial}{\partial x}+4(k-l)^{2}\right\} \\
& \times X_{k, l}(x)=0,
\end{aligned}
$$

where $Y_{m, n}(x)$ is written for arbitrary products $f_{m}(x) g_{n}(x)$ of solutions of (7.1) or their linear combinations. If we multiply the first equation with $X_{k, l}(x)$ and the second equation with $Y_{m, n}(x)$ and subtract the obtained equations then we find the following possible 
representation of the resulting equation

$$
\begin{aligned}
0= & \frac{\partial}{\partial x}\left\{X_{k, l}(x) Y_{m, n}^{(3)}(x)-X_{k, l}^{(1)}(x) Y_{m, n}^{(2)}(x)+X_{k, l}^{(2)}(x) Y_{m, n}^{(1)}(x)-X_{k, l}^{(3)}(x) Y_{m, n}(x)\right. \\
& \left.-2\left(2 x^{2}-(m+n+k+l+2)\right)\left\{X_{k, l}(x) Y_{m, n}^{(1)}(x)-X_{k, l}^{(1)}(x) Y_{m, n}(x)\right\}-4 x X_{k, l}(x) Y_{m, n}(x)\right\} \\
& +2(m+n-k-l)\left\{X_{k, l}(x) Y_{m, n}^{(2)}(x)+X_{k, l}^{(2)}(x) Y_{m, n}(x)\right\} \\
& +4\left((m-n)^{2}-(k-l)^{2}\right) X_{k, l}(x) Y_{m, n}(x) .
\end{aligned}
$$

By integration of this equation over the whole coordinate axis $R$ under the assumption that the products $X_{k, l}^{(r)}(x) Y_{m, n}^{(s)}(x)$ vanish for $x \rightarrow \pm \infty$ one finds

$$
\begin{aligned}
& (m+n-k-l) \int_{-\infty}^{+\infty} d x\left\{X_{k, l}(x) Y_{m, n}^{(2)}(x)+X_{k, l}^{(2)}(x) Y_{m, n}(x)\right\} \\
& +2\left\{(m-n)^{2}-(k-l)^{2}\right\} \int_{-\infty}^{+\infty} d x X_{k, l}(x) Y_{m, n}(x)=0 .
\end{aligned}
$$

The first of the integrals in (7.12) can be transformed by partial integration as follows

$$
\begin{aligned}
& \int_{-\infty}^{+\infty} d x\left\{X_{k, l}(x) Y_{m, n}^{(2)}(x)+X_{k, l}^{(2)}(x) Y_{m, n}(x)\right\} \\
= & \int_{-\infty}^{+\infty} d x\left\{-X_{k, l}^{(1)}(x)\left(\frac{\partial}{\partial x} Y_{m, n}(x)\right)+\left(\frac{\partial}{\partial x} X_{k, l}^{(1)}(x)\right) Y_{m, n}(x)\right\} \\
= & 2 \int_{-\infty}^{+\infty} d x\left(\frac{\partial}{\partial x} X_{k, l}^{(1)}(x)\right) Y_{m, n}(x) .
\end{aligned}
$$

From (7.13) and (7.12) one can derive different orthogonality relations. Consider the case

$$
m-n=k-l \equiv-j, \longrightarrow l=k+j, \quad n=m+j, \quad m+n-k-l=2(m-k) .
$$

Since the integral becomes nonvanishing only for $m-k=0$ one obtains

$$
\int_{-\infty}^{+\infty} d x\left(\frac{\partial}{\partial x} X_{k, k+j}^{(1)}(x)\right) Y_{m, m+j}(x)=\delta_{k, m} \int_{-\infty}^{+\infty} d x\left(\frac{\partial}{\partial x} X_{m, m+j}^{(1)}(x)\right) Y_{m, m+j}(x) .
$$

In particular, by choosing

$$
X_{k, l}^{(1)}(x)=h_{k}(x) g_{l}(x), \quad Y_{m, n}(x)=h_{m}(x) h_{n}(x),
$$

where $g_{l}(x)$ denotes the nonnormalizable solution (7.4) of Eq.(7.1) with parity $(-1)^{n+1}$ one finds from (7.15) with the help of (7.13)

$$
\int_{-\infty}^{+\infty} d x\left(\frac{\partial}{\partial x} h_{k}(x) g_{k+j}(x)\right) h_{m}(x) h_{m+j}(x)
$$




$$
\begin{aligned}
= & \delta_{k, m} \frac{1}{2} \int_{-\infty}^{+\infty} d x\left\{\left(\frac{\partial}{\partial x} h_{m}(x) g_{m+j}(x)\right) h_{m}(x) h_{m+j}(x)\right. \\
& \left.-h_{m}(x) g_{m+j}(x)\left(\frac{\partial}{\partial x} h_{m}(x) h_{m+j}(x)\right)\right\} \\
= & \delta_{k, m} \frac{1}{2} W\left(h_{m+j}(x), g_{m+j}(x)\right) \int_{-\infty}^{+\infty} d x h_{m}(x) h_{m}(x) \\
= & \delta_{k, m}
\end{aligned}
$$

where the special value of the Wronskian given in (4.29) was used. The special case $j=0$ of the orthogonality relations (7.17) can be obtained from the third-order differential equation (7.6) for $m=n$ in an easier way but for shortness we did not separately give its derivation.

Using the representation of $h_{m}(x)$ by Hermite functions given in (7.2) and the representation of $g_{n}(x)$ by functions of the parabolic cylinder given in (7.4) one obtains by differentiation of their product the following representation of the pattern functions (4.25)

$$
\begin{aligned}
F_{m, n}^{\prime \prime}(x)= & \frac{\partial}{\partial x}\left\{h_{m}(x) g_{n}(x)\right\} \\
= & \exp \left(-\frac{x^{2}}{2}\right) \sqrt{\frac{n !}{2^{m} m !}} i^{n}\left\{(n+1) H_{m}(x)\left(D_{-2-n}(i \sqrt{2} x)+(-1)^{n} D_{-2-n}(-i \sqrt{2} x)\right)\right. \\
& \left.+i \sqrt{2} m H_{m-1}(x)\left(D_{-1-n}(i \sqrt{2} x)-(-1)^{n} D_{-1-n}(-i \sqrt{2} x)\right)\right\} .
\end{aligned}
$$

This form of the pattern functions is not symmetric with respect to permutation of the indices $(m, n)$. It is identical with the pattern functions $F_{m, n}(x)$ in Eq.(4.25) only for $m \leq n+1$ as discussed in section 4 but can be taken as an equivalent pattern function for arbitrary $(m, n)$ due to their nonuniqueness as discussed in section 6. Furthermore, it is clear that, instead of (7.16), one can take for $X_{k, l}^{(1)}(x)$ the combination with exchange of the normalizable and nonnormalizable solutions of the wave equation, i.e.

$$
X_{k, l}^{(1)}(x)=g_{k}(x) h_{l}(x), \quad Y_{m, n}(x)=h_{m}(x) h_{n}(x)
$$

or linear combinations of them. In case of (7.19) we find as the equivalent pattern functions

$$
F_{m, n}^{\prime \prime \prime}(x)=\frac{\partial}{\partial x}\left\{g_{m}(x) h_{n}(x)\right\}=F_{n, m}^{\prime \prime}(x) .
$$

These pattern functions are identical with the pattern functions $F_{m, n}(x)$ considered in Eqs.(4.25) and (4.27) in the cases

$$
F_{m, n}^{\prime \prime}(x)=F_{m, n}(x), \quad m \leq n+1, \quad F_{m, n}^{\prime \prime \prime}(x)=F_{m, n}(x), \quad n \leq m+1,
$$

and are connected with the pattern functions $F_{m, n}^{\prime}(x)$ considered in the last section by

$$
F_{m, n}^{\prime}(x)=\frac{1}{2}\left(F_{m, n}^{\prime \prime}(x)+F_{n, m}^{\prime \prime}(x)\right) .
$$


Nevertheless, in all other cases of nonidentity one has completely equivalent pattern functions which can be used by same right as the pattern functions $F_{m, n}(x)$. As already mentioned, the pattern functions $F_{m, n}(x)$ possess the advantage that they vanish at infinity. In the special cases $m=n$ and $m=n \pm 1$ we obtained the same pattern functions by all considered methods.

The nonuniqueness of the pattern functions may have as the cause a certain redundancy in the information contained in the Wigner quasiprobability and in its Radon transform but it seems to be difficult to eliminate this redundancy. The pattern functions themselves play an auxiliary role and only the integrals (4.24) over the pattern functions leading to the Fock-state matrix elements possess an invariant meaning. The representations of the pattern functions in the form (7.18) or (7.20) is useful for computer calculations. In comparison to (4.27) they possess the advantage that one has to calculate here only a sum over the real or imaginary parts of two functions of the parabolic cylinder of imaginary argument instead of maximally $n+1$ such functions there. 


\section{Conclusion}

We introduced in the present paper the Radon transform of the Wigner quasiprobability in its more general canonical representation in comparison to the usual and considered the reconstruction of the density operator via the Fock-state matrix elements and via the normally ordered moments. The inverse two-dimensional Radon transformation was considered in detail because some moments seem to have been clarified in the literature about quantum tomography. The transformation properties of the Wigner quasiprobability and its Radon and Fourier transforms with regard to displacement and squeezing of an initial state are considered with many technical details. This has a more practical aspect, for example, for the calculation of the Radon transform of squeezed coherent states and squeezed-state excitations which becomes much easier by application of the derived formulae. The calculation of the pattern functions for the reconstruction of the Fock-state matrix elements of the density operator via the normally ordered moments leads to a new representation of these functions by convergent series over Hermite polynomials of even or odd order which provides an alternative for calculation and plotting of these functions.

We did not consider in the present paper, for example, the influence of imperfect measurements which lead to some smoothing of the Radon transform with the problem to reconstruct the density operator from these smoothed Radon transforms, a problem, now intensively discussed in the literature. Furthermore, we did not extend the considerations to multi-mode cases but many features can be probably translated to these more general cases in a simple way. We hope that our considerations are useful for the clarification of some principal problems connected with two-dimensional Radon transforms and its application in quantum optics.

\section{Acknowledgement}

The author likes to express his gratitude for valuable discussions and hints to V.P. Karassiov from Moscow, P.L. Knight from London, O.V. Man'ko and V.I. Man'ko from Moscow, T. Opatrný from Olomouc, M.G.A. Paris from Pavia and Th. Richter from Berlin. 


\section{Bibliography}

[1] M.G. Raymer, D.T.Smithey, M.Beck, and J.Cooper, Acta Phys. Polonica A 86, 71 (1994), ( Proceedings of the International Conference "Quantum Optics III", Szczyrk, Poland 1993 ).

[2] D.T. Smithey, M. Beck, J. Cooper, and M.G. Raymer, Phys. Rev. A 48, 3159 (1993).

[3] D.T. Smithey, M. Beck, J. Cooper, M.G. Raymer, and A. Faridani, Phys. Scr. 48, 35 (1993).

[4] D.T. Smithey, M. Beck, J. Cooper, M.G. Raymer, and A. Faridani, Phys.Rev.Lett. 70, 1244 (1993).

[5] K. Vogel and H. Risken, Phys.Rev. A 40, 2847 (1989).

[6] I.M. Gel'fand, M.I. Graev, and N.Ya. Vilenkin, Generalized Functions, Vol. 5: Integral Geometry and Representation Theory, Academic Press, New York 1966, Original in Russian: Fizmatgiz, Moscow 1962.

[7] S. Helgason, The Radon Transform, Birkhäuser, Boston 1980.

[8] H.H. Barrett, The Radon Transform and its Application, in: Progress in Optics (Ed. E. Wolf), Vol.21, p.217, North-Holland, Amsterdam 1984.

[9] G.M. D'Ariano, C. Macchiavello and M.G.A. Paris, Phys.Rev. A 50, 4298 (1994).

[10] G.M. D'Ariano, C. Macchiavello and M.G.A. Paris, Phys.Lett. A 195, 31 (1994).

[11] G.M. D’Ariano, Quantum Semiclass.Opt. 7, 693 (1995).

[12] U. Leonhardt, H. Paul, and G.M. D’Ariano, Phys.Rev. A 52, R1801 (1995).

[13] H. Paul, U. Leonhardt, and G.M. D’Ariano, Acta Phys.Slovaca 45, 261 (1995).

[14] U. Leonhardt, H. Paul, and G.M. D’Ariano, Phys.Rev. A 52, 4899 (1995).

[15] G.M. D’Ariano, C. Macchiavello and M.G.A. Paris, Opt.Comm. 129,6 (1996).

[16] M.G.A. Paris, Phys.Rev. A 53, 2658 (1996). 
[17] M.G.A. Paris, Opt.Comm. 124, 277 (1996).

[18] Th. Richter, Phys.Lett. A 211, 327 (1996).

[19] Th. Richter, and A. Wünsche, Phys.Rev. A 53, R 1974 (1996).

[20] U. Leonhardt, M. Munroe, T. Kiss, M.G. Raymer and Th. Richter, Opt.Comm. 127, 144 (1996).

[21] Th. Richter, and A. Wünsche, Acta Phys.Slovaca 46, 487 (1996).

[22] H. Kühn, D.-G. Welsch and W. Vogel, J.Mod.Opt. 41, 1607 (1994).

[23] H. Kühn, D.-G. Welsch and W. Vogel, Phys.Rev. A 51, 4240 (1995).

[24] Th. Richter, Phys.Rev. A 53, 53 (1996).

[25] A. Wünsche, Phys.Rev. A 54, 5291 (1996).

[26] T. Opatrný, D.-G. Welsch, S. Wallentowitz and W. Vogel, ( this special issue ).

[27] V. Bužek, G. Adam and G. Drobny̆, Phys.Rev. A 54, 804 (1996).

[28] S. Mancini, V.I. Manko and P. Tombesi, Quant.Semicl.Opt. 7, 615 (1995).

[29] S. Mancini, V.I. Manko and P. Tombesi, Phys.Lett. A 213, 1 (1996).

[30] E. Wigner, Phys.Rev. 40, 749 (1932).

[31] R.J. Glauber, Phys.Rev. 131, 2766 (1963).

[32] K.E. Cahill and R.J. Glauber, Phys.Rev. 177, 1857 and 1882 (1969).

[33] G.S. Agarwal and E. Wolf, Phys.Rev. D 2,2161 (1970).

[34] J. Peřina, Coherence of Light, Van Nostrand Reinhold, Amsterdam 1971.

[35] S.R. De Groot and L.G. Suttorp, Foundations of Electrodynamics, North-Holland, amsterdam 1972.

[36] V.J. Tatarski, Uspekhi Fiz. Nauk, 139, 587 (1983) ( in Russian ), Sov.Phys.Usp. 26, 311 (1983) ( in English ).

[37] M. Hillery, R.F. O'Connell, M.O. Scully and E.P. Wigner, Phys.Rep. 106, 121 (1984).

[38] A. Wünsche, Quantum Semiclass.Opt. 8, 343 (1996).

[39] I.M. Gel'fand and G.E. Shilov, Generalized Functions, Vol.1: Properties and Operations, Academic Press, New York 1964, Original in Russian: Fizmatgiz, Moscow 1958.

[40] A. Wünsche, Ann.Phys.(Leipzig) 1, 181 (1992). 
[41] A. Wünsche, Second International Workshop on Squeezed States and Uncertainty Relations, Moscow 1992, NASA Conference Publication 3219, p.277, Goddard Space Flight Center, Greenbelt, Maryland 1993.

[42] A.M. Perelomov, Generalized Coherent States and Their Applications, Springer, Berlin 1986.

[43] Y.S. Kim and M.E. Noz, Phase Space Picture of Quantum Mechanics, World Scientific, Singapore 1991.

[44] H. Weyl, The Classical Groups ( 2nd Edition ), Princeton University Press, Princeton ( New Jersey ) 1953.

[45] C.L. Mehta, J.Math.Phys. 18, 404 (1977).

[46] A. Ekert and P.L. Knight, Phys.Rev. A 42, 487 (1990).

[47] A. Ekert and P.L. Knight, Phys.Rev. A 43, 3934 (1991).

[48] D. Stoler, Phys.Rev. D 1, 3217 (1970).

[49] D. Stoler, Phys.Rev. D 4, 1925 (1970).

[50] H.P. Yuen, Phys.Rev. A 13, 2226 (1976).

[51] J.N. Hollenhorst, Phys.Rev. D 19, 1669 (1979).

[52] C.M. Caves, Phys.Rev. D 23, 1693 (1981).

[53] C.M. Caves, Phys.Rev. D 26, 1817 (1982).

[54] D.F. Walls, Nature 306, 141 (1983).

[55] R.A. Fisher, M.M. Nieto and V.D. Sandberg, Phys.Rev. D 291107 (1984).

[56] C.M.Caves and B.L. Schumaker, Phys.Rev. A 31, 3068 (1985).

[57] B.L. Schumaker and C.M. Caves, Phys.Rev. A 31, 3093 (1985).

[58] L. Mandel and E. Wolf, Optical Coherence and Quantum Optics, Cambridge University Press, New York 1995.

[59] K.A. Roy and C.L. Mehta, J.Mod.Opt. 39, 1619 (1992).

[60] E. Schrödinger, Ber.Kgl.Akad.Wiss.Berlin, 296 (1930).

[61] H.P. Robertson, Phys.Rev. 35, 667 (1930).

[62] V.V. Dodonov, V.I. Man'ko, Trudy FIAN im. Lebedeva ( Papers of the Lebedev Physical Institute of Academy of Science ), Vol. 183, p.5, Nauka, Moskva 1987. 
[63] A. Erdélyi, Higher Transcendental Functions, Vol. 2, McGraw-Hill, New York 1953.

[64] R.F. Bishop and A. Vourdas, Phys.Rev. A 50, 4488 (1994).

[65] A. Czirják, M.G. Benedict, acta physica slovaka 45, 341 (1995).

[66] A. Czirják, M.G. Benedict, J.Phys.A:Math.Gen. 28, 4599 (1995).

[67] G.M. D'Ariano, C. Macchiavello and N. Sterpi, preprint ( to be published in Phys.Rev. A ).

[68] A. Wünsche, Quant.Opt. 2, 453 (1990).

[69] C.T. Lee, Phys.Rev. A 46, 6097 (1992).

[70] U. Herzog, Phys.Rev. A 53, 2889 (1996).

[71] W.H. Louisell, Quantum Statistical Properties of Radiation, John Wiley, New York, 1973. ( Till to the beginning of the nineties I used mostly the older book of Louisell in its Russian translation which does not contain the corresponding formula ).

[72] A problem posed by U. Leonhardt to me was to prove or disprove that the first maxima and minima of $F_{n, n}(x)$ are exactly equal to the value $\pm F_{n, n}(0)$. This can be disproved numerically by applying the expansion in Hermite polynomials (6.3). For example, $F_{2,2}(0)=2$, first mimima $F_{2,2}( \pm 0.715045)=-2.00113$, first maxima $F_{2,2}( \pm 1.54108)=2.01617$.

[73] Fan Hong-yi, Liu Zu-wei and Ruan Tu-nan, Comm.in Theor.Phys. (Beijing), 3, 175 (1985).

[74] A. Wünsche, Acta Phys.Slovaca 46, 505 (1996). 